\title{
A non-Archimedean analogue of Teichmüller space and its tropicalization
}

\section{Martin Ulirsch ${ }^{1}$}

Accepted: 18 January 2021 / Published online: 29 May 2021

(c) The Author(s) 2021, corrected publication 2021

\begin{abstract}
In this article we use techniques from tropical and logarithmic geometry to construct a non-Archimedean analogue of Teichmüller space $\overline{\mathcal{T}}_{\mathrm{g}}$ whose points are pairs consisting of a stable projective curve over a non-Archimedean field and a Teichmüller marking of the topological fundamental group of its Berkovich analytification. This construction is closely related to and inspired by the classical construction of a nonArchimedean Schottky space for Mumford curves by Gerritzen and Herrlich. We argue that the skeleton of non-Archimedean Teichmüller space is precisely the tropical Teichmüller space introduced by Chan-Melo-Viviani as a simplicial completion of Culler-Vogtmann Outer space. As a consequence, Outer space turns out to be a strong deformation retract of the locus of smooth Mumford curves in $\overline{\mathcal{T}}_{\mathrm{g}}$.
\end{abstract}

Mathematics Subject Classification $14 \mathrm{~T} 20 \cdot 14 \mathrm{H} 10 \cdot 14 \mathrm{G} 22$

\section{Introduction}

Let $\mathrm{g} \geqslant 2$ and suppose for now that we are working over $\mathbb{C}$. Teichmüller space $\mathcal{T}_{\mathrm{g}, \mathbb{C}}$ is the universal cover of the moduli space $\mathcal{M}_{\mathrm{g}}$ of smooth curves of genus $\mathrm{g}$. It is a complex analytic space that functions as a fine moduli space of smooth curves $X$ (of genus $g$ ) together with a Teichmüller marking, that is a an equivalence $\phi: \pi_{1}(\mathrm{X}) \stackrel{\sim}{\longrightarrow} \Pi_{\mathrm{g}} 1$, where

$$
\Pi_{g}=\left\langle\alpha_{1}, \beta_{1}, \ldots, \alpha_{g}, \beta_{g} \mid\left[\alpha_{1}, \beta_{1}\right] \ldots\left[\alpha_{g}, \beta_{g}\right]=1\right\rangle
$$

\footnotetext{
1 Here $\pi_{1}(X)$ denotes the fundamental groupoid of $X$; since $X$ is connected, an equivalence $\phi: \pi_{1}(X) \stackrel{\sim}{\rightarrow} \Pi_{g}$ amounts to choosing an outer isomorphism class $\pi_{1}(X, x) \stackrel{\sim}{\longrightarrow} \Pi_{g}$ for one (and hence all) base points $x \in X$.
}

$凶$ Martin Ulirsch

ulirsch@math.uni-frankfurt.de

1 Institut für Mathematik, Goethe-Universität Frankfurt, 60325 Frankfurt am Main, Germany 
denotes the fundamental group of a fixed Riemann surface of genus $\mathrm{g}$.

The moduli space $\mathrm{M}_{\mathrm{g}}^{\text {trop }}$ of stable tropical curves of genus $\mathrm{g}$ is a combinatorial analogue of $\mathcal{M}_{\mathrm{g}}$ that captures the combinatorics of the dual complex of the Deligne-Mumford compactification $\overline{\mathcal{M}}_{\mathrm{g}}$ of $\mathcal{M}_{\mathrm{g}}$. A candidate for a tropical analogue of Teichmüller space is Outer space $\mathrm{CV}_{\mathrm{g}}$ in the sense of Culler-Vogtmann (see [28]), which arrived in the world of mathematics well before the recent spark in interest in tropical geometry (see [21, Section 5]). Outer space is a moduli space of metric graphs $\Gamma$ together with an equivalence $\phi: \pi_{1}(\Gamma) \stackrel{\sim}{\rightarrow} F_{g}$, where $F_{g}$ denotes the free group on g generators. In [26] Chan, Melo, and Viviani, building on [28], construct a tropical analogue $\mathrm{T}_{\mathrm{g}}^{\text {trop }}$ of Teichmüller space as a natural simplicial completion of Outer space $\mathrm{CV}_{\mathrm{g}}$ by allowing contractions of loops to vertices with integer weights.

Denote by $\mathcal{M}_{\mathrm{g}}^{\mathrm{an}}$ the non-Archimedean analytic space associated to $\mathcal{M}_{\mathrm{g}}$ (in the sense of Berkovich), where the base field is carrying the trivial absolute value. In [7] (based on earlier work in [78]) Abramovich, Caporaso, and Payne describe the connection between the algebraic and the tropical moduli space: they show that the moduli space of tropical curves is the target of a natural tropicalization map trop $: \mathcal{M}_{\mathrm{g}}^{\mathrm{an}} \rightarrow M_{\mathrm{g}}^{\text {trop }}$ that sends a smooth projective curve $X$ over a nonArchimedean field to its dual tropical curve $\Gamma_{X}$ (i.e. the minimal skeleton of $X^{\text {an }}$ decorated by certain vertex weights keeping track of the genus of components in the reduction). Moreover, it is shown that $\mathrm{M}_{\mathrm{g}}^{\text {trop }}$ may be naturally identified with the non-Archimedean skeleton of $\mathcal{M}_{\mathrm{g}}$ (defined with respect to the Deligne-Mumford compactification), so that trop $\mathrm{g}_{\mathrm{g}}$ is, in particular, a strong deformation retraction. Motivated by this beautiful story for $\mathcal{M}_{\mathrm{g}}$, one might be tempted to ask:

Which space tropicalizes to tropical Teichmüller space?

\section{A non-Archimedean Teichmüller space}

From now on let $\mathrm{K}$ be an algebraically closed non-Archimedean field (not necessarily carrying the trivial absolute value). In this article, inspired by the work of Gerritzen and Herrlich on a non-Archimedean analogue of Schottky space [31,32,36,38], we use methods from tropical and logarithmic geometry to construct a non-Archimedean Teichmüller space $\overline{\mathcal{T}}_{\mathrm{g}}$. Roughly speaking, a point in $\overline{\mathcal{T}}_{\mathrm{g}}$ is a stable projective curve $\mathrm{X}$ over a non-Archimedean extension $L$ of $K$ together with an equivalence

$$
\phi: \pi_{1}\left(\mathrm{X}^{\mathrm{an}}\right) \stackrel{\sim}{\longrightarrow} \mathrm{F}_{\mathrm{b}_{1}}
$$

where $b_{1}=b_{1}\left(X^{a n}\right)$ denotes the first Betti number of $X^{a n}$, thought of as a topological space.

Theorem A The non-Archimedean Teichmüller space $\overline{\mathcal{T}}_{\mathrm{g}}$ is an analytic DeligneMumford stack that is smooth, separated and without boundary.

We write $\mathcal{T}_{\mathrm{g}}$ for the locus of smooth curves in $\overline{\mathcal{T}}_{\mathrm{g}}$ and $\overline{\mathcal{T}}_{\mathrm{g}}^{\text {Mum }}$ as well as $\mathcal{T}_{\mathrm{g}}^{\text {Mum }}$ for the locus of stable or respectively smooth Mumford curves in $\overline{\mathcal{T}}_{\mathrm{g}}$. 


\section{Tropicalization}

Denote by $\overline{\mathrm{T}}_{\mathrm{g}}^{\mathrm{trop}}$ the canonical compactification of $\mathrm{T}_{\mathrm{g}}^{\mathrm{trop}}$ that parametrizes tropical curves $\Gamma$ where we allow the edge lengths to be $\infty$. There is a natural tropicalization map

$$
\operatorname{trop}_{\mathrm{g}}: \overline{\mathcal{T}}_{\mathrm{g}} \longrightarrow \overline{\mathrm{T}}_{\mathrm{g}}^{\text {trop }}
$$

that sends a pair $(X, \phi)$ as above to the dual tropical curve $\Gamma_{X}$ of $X$ together with the natural induced marking $\phi: \pi_{1}\left(\Gamma_{X}\right) \rightarrow F_{b_{1}}$ (using that $\Gamma_{X}$ is a strong deformation retract of $\left.X^{a n}\right)$. We show the following analogue of the main result of [7].

Theorem B The natural tropicalization map $\operatorname{trop}_{\mathrm{g}}: \overline{\mathcal{T}}_{\mathrm{g}} \longrightarrow \overline{\mathrm{T}}_{\mathrm{g}}^{\text {trop }}$ has a section that makes $\overline{\mathrm{T}}_{\mathrm{g}}^{\text {trop }}$ into a strong deformation retract of $\overline{\mathcal{T}}_{\mathrm{g}}$.

In particular, the restriction of trop ${ }_{g}$ to the locus $\mathcal{T}_{\mathrm{g}}$ of smooth curves in $\overline{\mathcal{T}}_{\mathrm{g}}$ induces a strong deformation retraction onto the (non-extended) tropical Teichmüller space $T_{g}$.

At this occasion, we point out that we prove Theorem B over any algebraically closed non-Archimedean field, contrary to the main result of [7] which is only proved over an algebraically closed field with the trivial absolute value. For this purpose we generalize in Sect. 4.5 below the construction of a Berkovich skeleton relative to a simple normal crossing divisor carried out in [34] to normal crossing divisors on smooth Deligne-Mumford stacks with good reduction. We also generalize [7, Theorem 1.2.1] to any algebraically closed non-Archimedean base fields in Theorem 4.4.

Restricting the contraction in Theorem B to the locus of smooth Mumford curves we find the following.

Corollary C The restriction of the tropicalization map to $\mathcal{T}_{\mathrm{g}}^{\mathrm{Mum}}$ makes the CullerVogtmann Outer space $\mathrm{CV}_{\mathrm{g}}$ into a strong deformation retract of $\mathcal{T}_{\mathrm{g}}^{\mathrm{Mum}}$.

Our construction of $\overline{\mathcal{T}}_{\mathrm{g}}$ uses the tropical construction of $\mathcal{T}_{\mathrm{g}}^{\text {trop }}$ and, in order to lift this to the algebraic world, it uses methods from logarithmic geometry in the sense of Fontaine-Kato-Illusie (see [44]) and, in particular, the theory of Artin fans, as developed in $[6,12,23,77]$. In fact, we define a logarithmic algebraic stack $\mathcal{T}_{\mathrm{g}}^{\text {log }}$ as the fibered product

$$
\mathcal{T}_{\mathrm{g}}^{\text {log }}=\mathcal{M}_{\mathrm{g}}^{\text {log }} \times{ }_{\mathcal{M}_{\mathrm{g}}^{\text {trop }}} \mathcal{T}_{\mathrm{g}}^{\text {trop }}
$$

along the natural tropicalization morphism trop $: \mathcal{M}_{\mathrm{g}}^{\text {log }} \rightarrow \mathcal{M}_{\mathrm{g}}^{\text {trop }}$ introduced in [23]. This way we obtain a smooth, universally closed, non-separated DeligneMumford stack $\underline{\mathcal{I}}_{\mathrm{g}}^{\text {log }}$ locally of finite type over $\mathbb{Z}$ in which the complement of the locus of smooth curves in $\underline{\mathcal{T}}_{\mathrm{g}}^{\mathrm{log}}$ is a divisor with simple normal crossings (over $\mathbb{Z}$ ). We then define the non-Archimedean Teichmüller space $\overline{\mathcal{T}}_{\mathrm{g}}$ as the analytic generic fiber of the base change of $\underline{\mathcal{I}}_{\mathrm{g}}^{\mathrm{log}}$ to the valuation ring $\mathrm{R}$ of $\mathrm{K}$. 


\section{Non-Archimedean Schottky space and its tropicalization}

In [30-32,36,38] Gerritzen and Herrlich construct a closely related non-Archimedean analogue $S_{\mathrm{g}}$ of Schottky space over the locus of Mumford curves in $\mathcal{M}_{\mathrm{g}}^{\mathrm{an}}$ (also see $[35,49]$ for the original complex construction and [62] for an incarnation of this in the language of formal schemes). They crucially use Mumford's non-Archimedean analogue of Schottky uniformization for maximally degenerate curves (as introduced in [56]; also see [29,52] for further details).

A point in $S_{g}$ is a is $P L_{2}(L)$-conjugacy class of a faithful representation $F_{g} \rightarrow$ $\mathrm{PGL}_{2}(\mathrm{~L})$ with discontinuous image for a non-Archimedean extension. Denote by $\Omega \subseteq \mathbb{P}_{\mathrm{L}}^{1}$, an the open set of ordinary points of the induced operation of $\mathrm{F}_{\mathrm{g}}$ on $\mathbb{P}_{\mathrm{L}}^{1}$, an . Then $\Omega / F_{g}$ is a Mumford curve $X^{\text {an }}$ and the induced equivalence $\pi_{1}(X) \stackrel{\sim}{\rightarrow} F_{g}$ is a marking in the above sense. So we have a natural morphism $S_{g} \rightarrow \overline{\mathcal{T}}_{g}$ whose image is the locus $\mathcal{T}_{\mathrm{g}}^{\text {Mum }}$ of smooth Mumford curves.

Denote by $\bar{S}_{g}$ the natural partial compactification of $S_{g}$ that extends their construction by faithful and discontinuous operations on trees of projective lines (constructed in [38] as a rigid-analytic space). Then the above morphism $\mathrm{S}_{\mathrm{g}} \rightarrow \overline{\mathcal{T}}_{\mathrm{g}}$ naturally extends to a morphism $\overline{\mathrm{S}}_{\mathrm{g}} \rightarrow \overline{\mathcal{T}}_{\mathrm{g}}$ whose image is the locus $\overline{\mathcal{T}}_{\mathrm{g}}^{\text {Mum }}$ of stable Mumford curves in $\overline{\mathcal{T}}_{\mathrm{g}}$.

Herrlich [40] was already aware that there is a natural "tropicalization map" from $\mathrm{S}_{\mathrm{g}}$ to Culler-Vogtmann Outer space $\mathrm{CV}_{\mathrm{g}}$. In this article we expand on this realization and recover his map as the composition $\mathrm{S}_{\mathrm{g}} \rightarrow \mathcal{T}_{\mathrm{g}}^{\text {Mum }} \rightarrow \mathrm{CV}_{\mathrm{g}}$. We refer the reader to the upcoming [63] for a detailed examination of the relationship between $S_{\mathrm{g}}$ and $\mathcal{T}_{\mathrm{g}}^{\mathrm{Mum}}$.

\section{The quotient by Out $\left(F_{g}\right)$}

Consider the natural forgetful map $\overline{\mathcal{T}}_{\mathrm{g}} \rightarrow \overline{\mathcal{M}}_{\mathrm{g}}$ that forgets the marking. There is a natural operation of $\operatorname{Out}\left(\mathrm{F}_{\mathrm{g}}\right)$ on $\overline{\mathcal{T}}_{\mathrm{g}}$ with respect to which the forgetful map $\overline{\mathcal{T}}_{\mathrm{g}} \rightarrow \overline{\mathcal{M}}_{\mathrm{g}}$ is invariant. The quotient stack $\left[\overline{\mathcal{M}}_{\mathrm{g}} / \operatorname{Out}\left(\mathrm{F}_{\mathrm{g}}\right)\right]$ is not isomorphic to $\overline{\mathcal{M}}_{\mathrm{g}}^{\mathrm{an}}$, since nonArchimedean curves with non-maximal reduction will always have stabilizer groups that are not present in $\overline{\mathcal{M}}_{\mathrm{g}}^{\mathrm{an}}$. We, however, have the following weaker Theorem $\mathrm{D}$.

Theorem D The relative coarse quotient $\overline{\mathcal{T}}_{\mathrm{g}} / \overline{\mathcal{M}}_{\mathrm{g}}^{\text {an }} \operatorname{Out}\left(\mathrm{F}_{\mathrm{g}}\right)$ over $\overline{\mathcal{M}}_{\mathrm{g}}^{\mathrm{an}}$ is equal to $\overline{\mathcal{M}}_{\mathrm{g}}^{\mathrm{an}}$.

Here the relative coarse quotient $\overline{\mathcal{T}}_{\mathrm{g}} / \overline{\mathcal{M}}_{\mathrm{g}}^{\text {an }} \operatorname{Out}\left(\mathrm{F}_{\mathrm{g}}\right)$ is the relative coarse moduli space of $\left[\overline{\mathcal{T}}_{\mathrm{g}} / \operatorname{Out}\left(\mathrm{F}_{\mathrm{g}}\right)\right]$ over $\overline{\mathcal{M}}_{\mathrm{g}}^{\mathrm{an}}$, i.e. the morphism $\left[\overline{\mathcal{T}}_{\mathrm{g}} / \operatorname{Out}\left(\mathrm{F}_{\mathrm{g}}\right)\right] \rightarrow \overline{\mathcal{M}}_{\mathrm{g}}^{\mathrm{an}}$ is initial among all factorizations

$$
\left[\overline{\mathcal{T}}_{\mathrm{g}} / \operatorname{Out}\left(\mathrm{F}_{\mathrm{g}}\right)\right] \longrightarrow \mathcal{X} \longrightarrow \overline{\mathcal{M}}_{\mathrm{g}}^{\mathrm{an}}
$$

such that $\mathcal{X} \rightarrow \overline{\mathcal{M}}_{\mathrm{g}}^{\text {an }}$ is representable (see [9, Theorem 3.1] for the concept of relative coarse moduli spaces in the algebraic category). In our case, this means that 
$\overline{\mathcal{T}}_{\mathrm{g}} / \overline{\mathcal{M}}_{\mathrm{g}}^{\text {an }} \operatorname{Out}\left(\mathrm{F}_{\mathrm{g}}\right)$ up to natural equivalence is the only analytic stack that gives rise to such a factorization.

For a stable vertex-weighted graph $\mathrm{G}$ denote by $\overline{\mathcal{T}}_{\mathrm{G}}$ and $\overline{\mathcal{U}}_{\mathrm{G}}$ the affinoid domains of $\overline{\mathcal{T}}_{\mathrm{g}}$ and $\overline{\mathcal{M}}_{\mathrm{g}}^{\text {an }}$ respectively that parametrize stable curves of genus $\mathrm{g}$ for whom the underlying graph of the dual tropical curve is equal to $G$. Then Theorem $D$ means that we have an equivalence

$$
\overline{\mathcal{U}}_{\mathrm{G}} \simeq\left[\overline{\mathcal{T}}_{\mathrm{G}} / \operatorname{Out}\left(\mathrm{F}_{\mathrm{b}_{1}(\mathrm{G})}\right)\right]
$$

for every stable vertex-weighted graph $G$ with Betti-number $b_{1}(G)=h^{1}(G)$.

As a consequence of Theorem $\mathrm{D}$, we finally obtain:

Corollary $\mathrm{E}$ The coarse moduli space of the quotient $\left[\overline{\mathcal{T}}_{\mathrm{g}} / \operatorname{Out}\left(\mathrm{F}_{\mathrm{g}}\right)\right]$ is naturally isomorphic to the coarse moduli space $\overline{\mathrm{M}}_{\mathrm{g}}^{\mathrm{an}}$ of $\overline{\mathcal{M}}_{\mathrm{g}}^{\mathrm{an}}$.

Here the compatibility of forming coarse moduli space with taking analytification follows from [27, Theorem 1.2.1 and 1.2.2] on the analytification of algebraic spaces and étale equivalence relations.

\section{Complements and remarks}

1. Let $g, n \geqslant 0$ such that $2 g-2+n>0$. Our construction admits an immediate generalization to the case curves with marked points (as introduced in [48]). In fact, one may construct a non-Archimedean Teichmüller space $\overline{\mathcal{T}}_{\mathrm{g}, \mathrm{n}}$ parametrizing smooth projective curves over a non-Archimedean extension $L$ together with $n$ distinct marked points $p_{1}, \ldots, p_{n} \in X(L)$ and a fixed equivalence $\pi_{1}\left(X^{a n}\right) \simeq$ $\mathrm{F}_{\mathrm{b}_{1}(\mathrm{X})}$ as the fiber product

$$
\overline{\mathcal{T}}_{\mathrm{g}, \mathrm{n}}=\overline{\mathcal{T}}_{\mathrm{g}} \times \overline{\mathcal{M}}_{\mathrm{g}}^{\mathrm{an}} \overline{\mathcal{M}}_{\mathrm{g}, \mathrm{n}}^{\mathrm{an}}
$$

Analogues of the above results for $\overline{\mathcal{T}}_{\mathrm{g}, \mathrm{n}}$ immediately follow from this description and the natural forgetful map $\overline{\mathcal{T}}_{\mathrm{g}, \mathrm{n}+1} \rightarrow \overline{\mathcal{T}}_{\mathrm{g}, \mathrm{n}}$ functions as a universal curve.

2. In [54] Mochizuki develops another approach to the non-Archimedean uniformization of $\mathcal{M}_{\mathrm{g}}$ that goes by the name $p$-adic Teichmüller theory (also see [55]). As explained [55, Section 1.1] this is based on a p-adic analogue of Fuchsian uniformization via so-called indigenous bundles, while our approach is essentially based on Mumford's non-Archimedean analogue of Schottky uniformization (as introduced in [56]). Mochizuki, in particular, argues that Mumford's uniformization "does not strongly depend on the prime p", since e.g. Frobenius automorphisms play no significant role. The fact that our space $\underline{\mathcal{I}}_{g}^{\text {log }}$ is actually defined over $\mathbb{Z}$ is another incarnation of this heuristic.

3. In this article we only use the topological fundamental group of a Berkovich analytic curve $X^{a n}$. So, for example, for curves with good reduction our construction introduces nothing new. The author believes there should be a "better" analogue of Teichmüller space over the $p$-adic numbers that uses a different analogue of the 
fundamental group of $X^{\mathrm{an}}$, e.g. the tempered fundamental group of André [8]. In [71, Chapter III] Scholze constructs a p-adic version of Siegel upper half plane in the framework of perfectoid spaces. A promising direction of future research could be to use his methods to try to construct a $p$-adic analogue of Teichmüller space. We refer the reader to [68] for further indications towards an abelian version of such a construction.

4. In an upcoming project [63] Poineau and Turchetti generalize Gerritzen-Herrlich Schottky space $S_{g}$ to a hybrid (Archimedean and non-Archimedean) analytic space over Spec $\mathbb{Z}$. Its fiber over a non-Archimedean place of $\mathbb{Z}$ is exactly the nonArchimedean Gerritzen-Herrlich Schottky space $S_{g}$ and over the Archimedean place it is the complex-analytic Schottky space $S_{g, \mathbb{C}}$ (as in $\left.[35,49]\right)$. An interesting trajectory for future research would be to develop a theory of hybrid analytic stacks in order to study the hybrid analytification of $\underline{\mathcal{I}}_{\mathrm{g}}^{\mathrm{log}}$, to compare it with the Poineau-Turchetti construction in [63], and to study its tropicalization from both an Archimedean and a non-Archimedean perspective.

5. In their project [63], Poineau and Turchetti, in particular, construct a uniformization of the universal Mumford curve. In our framework, we can recover this as follows: Denote by $\Omega_{\mathrm{g}}^{\text {trop }}$ the tropical moduli stack of tuples $((\widetilde{\Gamma}, l), f: \widetilde{\Gamma} \rightarrow \Gamma)$ consisting of a stable tropical curve $(\widetilde{\Gamma}, l)$ with one marked leg together with a length-preserving morphism $\mathrm{f}: \widetilde{\Gamma}^{s t} \rightarrow \Gamma$ from the stabilization $\widetilde{\Gamma}^{s t}$ of $\widetilde{\Gamma}$ (without the marked leg) to a stable tropical curve $\Gamma$ of genus $g$ that is a universal cover of the underlying weighted graphs. There is a natural morphism $\Omega_{\mathrm{g}}^{\text {trop }} \rightarrow \mathcal{M}_{\mathrm{g}, 1}^{\text {trop }}$ that is given by sending $((\widetilde{\Gamma}, l), f)$ to $(\Gamma, f(l))$ and we may again build its logarithmic analogue as the fibered product

$$
\Omega_{\mathrm{g}}^{\log }=\mathcal{M}_{\mathrm{g}, 1}^{\log } \times{ }_{\mathcal{M}_{\mathrm{g}, 1}^{\text {trop }}} \Omega_{\mathrm{g}}^{\text {trop }}
$$

If we apply the Raynaud generic fiber to $\underline{\Omega}_{\mathrm{g}}^{\mathrm{log}}$, we find a non-Archimedean analytic stack $\Omega_{\mathrm{g}}$ together with morphisms

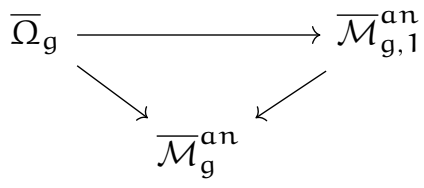

that functions as a non-Archimedean uniformization of the whole universal curve $\overline{\mathcal{M}}_{\mathrm{g}, 1}^{\mathrm{an}} \rightarrow \overline{\mathcal{M}}_{\mathrm{g}}^{\mathrm{an}}$. So there is a natural operation of $\mathrm{F}_{\mathrm{g}}$ on $\bar{\Omega}_{\mathrm{g}}$ such that the relative coarse quotient $\bar{\Omega}_{\mathrm{g}} / \overline{\mathcal{M}}_{\mathrm{g}, 1}^{\mathrm{an}} \mathrm{F}_{\mathrm{g}}$ is isomorphic to $\overline{\mathcal{M}}_{\mathrm{g}, 1}^{\mathrm{an}}$ and the restriction of (1) to a smooth Mumford curve $X$ in $\mathcal{M}_{g}^{a n}$ is exactly the presentation $X^{a n} \simeq \Omega / F_{g}$ of $X^{a n}$ as a quotient of an open subset of $\mathbb{P}^{1 \text {, an }}$ by a Schottky group (as in [56]). In [43] Ichikawa constructs the universal deformation of a Mumford curve via Schottky groups in order to study the extension problem for Teichmüller modular 
forms. His construction of a universal deformation may be recovered by considering the formal fiber of $\bar{\Omega}_{\mathrm{g}}$ over a Mumford curve in $\overline{\mathcal{M}}_{\mathrm{g}}$.

6. Of course it is natural to wonder whether there is also a connection between the classical complex analytic Teichmüller space $\mathcal{T}_{\mathrm{g}, \mathbb{C}}$ and its tropical analogue $\mathrm{T}_{\mathrm{g}}^{\text {trop }}$. In [33], Gerritzen and Herrlich construct a smooth compactification $\overline{\mathcal{S}}_{\mathrm{g}, \mathbb{C}}$ of Archimedean Schottky space, whose boundary combinatorics around the strata of maximally degenerate Riemann surfaces captures exactly the combinatorics of Culler-Vogtmann Outer space $\mathrm{CV}_{\mathrm{g}}$. We expect that a careful recasting of the extended Schottky spaces $\overline{\mathcal{S}}_{\mathrm{g}, \mathbb{C}}$ in terms of logarithmic geometry would allow us to construct a tropicalization morphism $\mathcal{S}_{\mathrm{g}, \mathbb{C}}^{\mathrm{log}} \rightarrow \mathcal{T}_{\mathrm{g}}^{\text {trop }}$ in the category of logarithmic $\mathbb{C}$-analytic stacks.

In [37] Herrlich uses this compactification (and Abikoff's bordification of Teichmüller space [4]) to construct a partial compactification of Teichmüller space $\overline{\mathcal{T}}_{\mathrm{g}, \mathbb{C}}$ as a complex ringed space that admits an operation of the mapping class group so that the coarse moduli space of $\left[\overline{\mathcal{T}}_{\mathrm{g}, \mathbb{C}} / \mathrm{MCG}_{\mathrm{g}}\right]$ is isomorphic to the coarse moduli space $\bar{M}_{\mathrm{g}, \mathbb{C}}$ over $\mathbb{C}$. For every symplectic homomorphism $\psi: \Gamma_{\mathrm{g}} \rightarrow \mathrm{F}_{\mathrm{g}}$ there is an open subset $\overline{\mathcal{T}}_{\mathrm{g}}(\psi) \subseteq \overline{\mathcal{T}}_{\mathrm{g}, \mathbb{C}}$ (containing $\mathcal{T}_{\mathrm{g}, \mathbb{C}}$ as an open and dense subset) that admits a morphism $\overline{\mathcal{T}}_{\mathrm{g}}(\psi) \rightarrow \overline{\mathcal{S}}_{\mathrm{g}, \mathbb{C}}$ (given by composing the Teichmüller marking with $\psi$ ). Composing such a morphism with the (conjectural) tropicalization morphism from above, would provide us with a procedure to tropicalize Teichmüller space $\mathcal{T}_{\mathrm{g}, \mathbb{C}} \subseteq \mathcal{T}_{\mathrm{g}}(\psi)$.

7. Our article is by no means the first instance where moduli functors combine both algebraic/analytic and tropical data. In particular, we refer the reader to the following works:

- to [79,81] for a non-Archimedean counting of holomorphic cylinders on CalabiYau surfaces, to [69,70] for a logarithmic/tropical reinterpretation of the VakilZinger blow of moduli spaces of elliptic stable maps on toric varieties, and to [66] and [67] for an approach to a degeneration formula [3] and a product formula [39] in logarithmic Gromov-Witten theory;

- to [58,59], to [41,42,50], as well as to [11] and [53] for an approach towards constructing a compactification of the universal Jacobian and a resolution of the universal Abel-Jacobi map;

- to [14] for a construction of a compactification of a strata of abelian differentials using combinatorial data which may be translated into tropical language expanding on [57]; and

- to [47] for a modular interpretation of toroidal compactifications of the moduli space $\mathcal{A}_{\mathrm{g}}$ of principally polarized complex abelian varieties.

Incarnations of the identification between the tropical analogue of a moduli space and its non-Archimedean skeleton, beyond [7], have also appeared in [25] for Hurwitz spaces, in [74] for the moduli space of weighted stable curves, in [65] for rational curves on toric varieties, in [24] for moduli spaces of spin curves, in [57] for moduli spaces of effective divisors, and in [10,22] for the universal Jacobian, as well as in [19] for Jacobians, in [20] for symmetric powers, and in [51] for Prym varieties. 


\section{Tropical moduli stacks}

In this section we introduce the basic terminology that we need to study tropical moduli spaces and introduce the moduli space of tropical curves. Our presentation is distilled from $[7,23,77]$.

\subsection{Cones and cone complexes}

An (abstract) rational polyhedral cone is a tuple $(\sigma, M)$ consisting of a topological space $\sigma$ together with a finitely generated free subgroup $M$ of the group of continuous real-valued functions on $\sigma$ such that the evaluation map $\sigma \rightarrow \operatorname{Hom}(M, \mathbb{R})$ induces a homeomorphism between $\sigma$ and a strictly convex rational polyhedral cone in $N_{\mathbb{R}}:=$ $\operatorname{Hom}(M, \mathbb{R})$ (i.e. a finite intersection of rational half-spaces). A morphism of rational polyhedral cones $(\sigma, M) \rightarrow\left(\sigma^{\prime}, M^{\prime}\right)$ is a continuous map $\sigma \rightarrow \sigma^{\prime}$ that pulls back $M^{\prime}$ to $M$. We usually drop the reference to $M$ from our notation.

The dual monoid of a rational polyhedral cone $\sigma$ is the submonoid $S_{\sigma}$ of those functions in $M$ that are non-negative on $\sigma$. We may recover $\sigma$ from $S_{\sigma}$ via the identification $\sigma=\operatorname{Hom}\left(S_{\sigma}, \mathbb{R}_{\geqslant 0}\right)$. In fact, the association $(\sigma, M) \mapsto S_{\sigma}$ defines an equivalence of between the category RPC of rational polyhedral cones and the category of finitely generated and integral (i.e. fine), saturated, and sharp monoids. A face $\tau$ of $\sigma$ is a subset along which a function $u \in S_{\sigma}$ vanishes. It naturally carries the structure of a rational polyhedral cone and the dual monoid of $\tau$ is given by the quotient $S_{u} / S_{u}^{*}$, where $S_{u}$ denotes the localization

$$
S_{\sigma}=\left\{s-k u \mid s \in S_{\sigma} \text { and } k \in \mathbb{N}\right\}
$$

and $S_{u}^{*}$ is the submonoid of units in $S_{\mathfrak{u}}$. A face morphism is a morphism $\tau \rightarrow \sigma$ that induces an isomorphism between $\tau$ and a face of $\sigma$. Notice here that, in particular, all automorphisms of a rational polyhedral cone are face morphisms. We say that a face morphism is proper if it is not an isomorphism.

Definition 1.1 A (rational polyhedral) cone complex is $\Sigma$ is a topological space $|\Sigma|$ together with a collection of closed subsets $\sigma_{i}$ (with $i \in$ I) that cover $|\Sigma|$ and carry the structure of a rational polyhedral cone subject to the following axioms:

(1) The intersection $\sigma_{i} \cap \sigma_{j}$ is a (necessarily finite) union of faces of each $\sigma_{i}$ and $\sigma_{j}$.

(2) For every face $\tau$ of $\sigma_{i}$ there is $j \in I$ such that $\sigma_{j}=\tau$.

(3) A subset $A \subseteq|\Sigma|$ is closed if and only if $A \cap \sigma_{i}$ is closed for all $i \in I$.

In other words, a cone complex $\Sigma$ is a colimit (in the category of topological spaces) over a poset of cones connected by face morphisms (see [23, Section 2.1] for details). A morphism $\Sigma \rightarrow \Sigma^{\prime}$ of cone complexes is a continuous map $|\Sigma| \rightarrow\left|\Sigma^{\prime}\right|$ such that for every cone $\sigma_{i} \subseteq \Sigma$ there is a cone $\sigma_{j}^{\prime} \subseteq \Sigma^{\prime}$ such that $f$ factors through a morphism $\sigma_{i} \rightarrow \sigma_{j}^{\prime}$ in RPC. We denote the category of rational polyhedral cone complexes by RPCC.

A morphism $f: \Sigma \rightarrow \Sigma^{\prime}$ is said to be strict, if the induced map $\sigma_{i} \rightarrow \sigma_{j}^{\prime}$ is a face morphism. Denote the class of strict morphism by $\mathbb{P}_{\text {strict }}$. By [23], strict 
morphisms define a subcanonical Grothendieck topology $\tau_{\text {strict }}$ on RPCC, and the triple (RPCC, $\left.\tau_{\text {strict }}, \mathbb{P}_{\text {strict }}\right)$ defines a geometric context in the sense of [23, Section 1].

Remark 1.2 The category RPCC is naturally equivalent to the category of (fine and saturated) Kato fans, an incarnation of the geometry over the field with one element introduced in [45] (see [75, Prop. 3.7]). Strict morphisms hereby correspond exactly to local isomorphism of Kato fans.

\subsection{Cone stacks and combinatorial cone stacks}

Let $\Sigma$ be a cone complex. We identify $\Sigma$ with its associated functor of points

$$
\begin{aligned}
h_{\Sigma}: \mathbf{R P C C} & \longrightarrow \text { Sets } \\
\Sigma^{\prime} & \longmapsto \operatorname{Hom}\left(\Sigma^{\prime}, \Sigma\right)
\end{aligned}
$$

so that, by Yoneda's Lemma, we can think of $\Sigma$ as both a presheaf and as a category fibered in groupoids RPCC/ $\Sigma$ over RPCC. We, in particular, say that a category fibered in groupoids over RPCC is representable by $\Sigma$ if it is equivalent to $\mathbf{R P C C} / \Sigma$.

Definition 1.3 A (rational polyhedral) cone stack is a category $\mathcal{C}$ fibered in groupoids over RPCC that is a stack with respect to the strict topology $\tau_{\text {strict }}$ that fulfils the following two axioms:

(i) the diagonal $\Delta: \mathcal{C} \longrightarrow \mathcal{C} \times \mathcal{C}$ is representable by cone complexes; and

(ii) there is a cone complex $\Sigma$ and a (necessarily representable) morphism $\Sigma \rightarrow \mathcal{C}$ that is strict and surjective.

The map $\Sigma \rightarrow \mathcal{C}$ is also called a chart of $\mathcal{C}$. Cone stacks naturally form a 2 -category. Its morphisms are morphism of categories over RPCC. The usual techniques for working with stacks apply to this situation as well. In particular, given a strict surjective groupoid object $\mathrm{R} \rightrightarrows \mathrm{U}$ in $\mathbf{R P C C}$ the quotient $[\mathrm{U} / \mathrm{R}]$ is cone stack, and, conversely, given a chart $\mathrm{U} \rightarrow \mathcal{C}$ of a cone stack $\mathcal{C}$, the fiber product $\mathrm{U} \times_{\mathcal{C}} \mathrm{U}$ is representable by a cone complex $\mathrm{R}$ so that $\mathrm{R} \rightrightarrows \mathrm{U}$ defines a strict and surjective groupoid object in $\mathbf{R P C C}$ and we have a natural equivalence $[\mathrm{U} / \mathrm{R}] \simeq \mathcal{C}$.

In $[23$, Section 2.2] the authors have introduced a combinatorial characterization of cone stacks.

Definition 1.4 A combinatorial cone stack is a category fibered in groupoids over RPC $^{f}$, the category of rational polyhedral cones with only face morphisms.

By [23], there is a natural equivalence between the 2-category of cone stacks and the 2-category of combinatorial cone stacks. Given a cone stack $\mathcal{C}$ an object in the associated combinatorial cone stack is a strict morphism $\sigma \rightarrow \mathcal{C}$ from a rational polyhedral cone $\sigma$ into $\mathcal{C}$ and a morphism is a commuting diagram

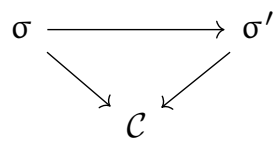


which is automatically a face morphism. Conversely, given a combinatorial cone stack $\mathcal{C}^{\mathrm{comb}}$, the associated cone $\operatorname{stack} \mathcal{C}$ is the unique stack over (RPCC, $\left.\tau_{\text {strict }}\right)$ whose fiber over a cone $\sigma$ is the groupoid $\operatorname{HOM}\left(\sigma, \mathcal{C}^{\text {comb }}\right)$.

\subsection{Coarse moduli spaces and generalized cone complexes}

In the following we write FAN, when we think of $\mathbf{R P C}{ }^{f}$ as a category fibered in groupoids over itself. So for every cone stack $\mathcal{C}$, there is a tautological morphism $\mathcal{C}^{\text {comb }} \rightarrow$ FAN.

Definition 1.5 A cone stack $\mathcal{C}$ is said to have faithful monodromy, if the tautological morphism $\mathcal{C}^{\mathrm{comb}} \rightarrow$ FAN is representable.

In other words, this means that morphisms in $\mathcal{C}^{\mathrm{comb}}$ are all actual face morphism. Alternatively, one may also think of cone stacks with faithful monodromy as (relative) sheaves over the category FAN.

Proposition 1.6 Let $\mathcal{C}$ be a cone stack. Then there is a cone stack $\mathrm{C}$ with faithful monodromy together with a strict morphism $\mathcal{C} \rightarrow \mathcal{C}$ that is initial among all strict morphisms from $\mathcal{C}$ into cone stacks with faithful monodromy.

In other words $\mathcal{C} \rightarrow \mathrm{C} \rightarrow$ FAN is the initial factorization of the tautological morphisms $\mathcal{C} \rightarrow$ FAN such that $\mathrm{C} \rightarrow$ FAN is representable. So the $\mathcal{C} \rightarrow C$ is the relative coarse moduli space of $\mathcal{C}$ over FAN. In a way, the morphism $\mathcal{C} \rightarrow C$ plays the role of the morphism of an algebraic stack to its coarse moduli space. Therefore we refer to $\mathcal{C} \rightarrow \mathrm{C}$ and, in a slight abuse of notation, to $\mathrm{C}$ as the coarse moduli space of $\mathcal{C}$.

Proof of Proposition 1.6 Using the identification of cone stacks with Artin fans from [23, Theorem 3] (see also Sect. 3.3 below), Proposition 1.6 is a special case of [6, Propostion 3.1.1]. The combinatorial cone stack $C^{\text {comb }}$ has the same objects as $\mathcal{C}$. For two objects $\alpha$ and $\beta$ in $\mathcal{C}^{\text {comb }}$ over cones $\sigma$ and $\tau$ respectively, the morphisms in $C^{c o m b}$ are the image of $\operatorname{Hom}_{\mathcal{C}}(\alpha, \beta)$ in $\operatorname{Hom}(\sigma, \tau)$. We may now easily verify that $\mathrm{C}^{\mathrm{comb}}$ fulfils the axioms of a category fibered in groupoids over $\mathbf{R P C}{ }^{f}$, that it has faithful monodromy, and that $\mathcal{C}^{\mathrm{comb}} \rightarrow \mathrm{C}^{\mathrm{comb}}$ is initial among all strict morphisms to combinatorial cone stacks with faithful monodromy.

Cone stacks are a refinement of the notion of a generalized cone complexes, as introduced in [7] as a generalization of cone complexes.

Definition 1.7 [7] A generalized cone complex as a topological space together with a presentation as a colimit of a diagram of (not necessarily proper) face morphisms.

A morphism of generalized cone complexes is a continuous map that locally factors through a morphism in RPC. The combinatorial cone stack associated to a cone stack with faithful monodromy defines a generalized cone complex. Conversely, adding all 
faces and pullback of invariant automorphisms, the defining diagram of a generalized cone complex generates a combinatorial cone stack. In fact, we have a natural equivalence (of 1-categories)

$\{$ cone stacks with faithful monodromy $\} /\{2$-isomorphisms $\} \simeq\{$ generalized cone complexes $\}$.

\subsection{Graphs}

Expanding on [72] and [23], a graph $\mathrm{G}$ consists of a set $\mathrm{X}=\mathrm{X}(\mathrm{G})$ together with an idempotent root map $r: X \rightarrow X$ and an involution $i: X \rightarrow X$ such that $r=i \circ r$. We refer to the set $V(G)=r(X)$ as the set of vertices of $G$ and to its complement as the set $H(G)$ of half edges of $G$. An element in the quotient $H(G) / i$ is of the form $[h \sim i(h)]$ for an half-edge $h$ of $G$; we refer to $[h \sim i(h)]$ as a finite edge when $h \neq i(h)$ and otherwise as a leg. So the quotient $X / i$ decomposes as a disjoint union $V(G) \sqcup E(G) \sqcup L(G)$, where $E(G)$ is the set of finite edges and $L(G)$ is the set of legs.

We say that a graph $G$ is finite, if $X(G)$ is finite. An order on an edge $e=[h \sim i(h)]$ is the choice of a relation $h<i(h)$ or $h>i(h)$. For an ordered edge $e=[h<i(h)]$, we write $\bar{e}$ for the same edge with the reverse order $[h>i(h)]$. A path $\gamma$ in a graph $G$ is a tuple $\left(v_{0} ; e_{1}, \ldots, e_{n}\right)$ consisting of a vertex $v_{0}$ and ordered edges $e_{i}$ of $G$ such that $r\left(\widetilde{h}_{1}\right)=v_{0}$ and for every ordered edge $e_{i}=\left[h_{i}<\widetilde{h}_{i}\right]$ (with $1 \leqslant i \leqslant n$ ) we have $r\left(h_{i}\right)=r\left(\widetilde{h}_{i+1}\right)$. We write $v_{i}$ for the vertex $r\left(h_{i}\right)=r\left(i\left(h_{i+1}\right)\right)$ and $v_{n}=r\left(h_{n}\right)$. Note that this notation is consistent, since $v_{0}=r\left(i\left(h_{1}\right)\right)$, and that the specification of $v_{0}$ in a path is only necessary, when the tuple $\left(e_{1}, \ldots, e_{n}\right)$ is empty; in this case the tuple $\left(v_{0}, \emptyset\right)$ refers to the constant path at $v_{0}$ and will be abbreviated by $v_{0}$. Given two paths $\gamma=\left(v_{0} ; e_{1}, \ldots, e_{n}\right)$ and $\gamma^{\prime}=\left(v_{0}^{\prime} ; e_{1}^{\prime}, \ldots, e_{n^{\prime}}^{\prime}\right)$ with $v_{n}=v_{0}^{\prime}$, we write

$$
\gamma \circ \gamma^{\prime}=\left(v_{0} ; e_{1}, \ldots, e_{n}, e_{1}^{\prime}, \ldots, e_{n^{\prime}}^{\prime}\right)
$$

for the concatenation of $\gamma$ and $\gamma^{\prime}$. We say that $\mathrm{G}$ is connected, if for any two vertices $v, w$ there is a path $\gamma$ in $G$ with $v_{0}=v$ and $v_{n}=w$. A path is said to be closed if $v_{0}=v_{n}$; in this case we refer to $v_{0}=v_{n}$ as the base point of the path $\gamma$. From now on we assume that all our graphs are connected.

A vertex weight on a graph $G$ is a function $h: V(G) \rightarrow \mathbb{Z}_{\geqslant 0}$; a marking $m$ on the set of legs of $G$ is a choice of total order on L. Whenever convenient we drop the reference to $h$ and $m$ from our notation and denote a weighted (marked) graph simply by $\mathrm{G}$.

The valence $\operatorname{val}(v)$ of a vertex $v$ of $G$ is the number of half edges $h$ with $r(f)=v$. A weighted marked graph is said to be stable, if for all vertices $v \in V(G)$ we have

$$
2 h(v)-2+\operatorname{val}(v)>0
$$

The genus $\mathrm{g}(\mathrm{G})$ of $\mathrm{G}$ is defined to be $\mathrm{b}_{1}(\mathrm{G})+\sum_{v \in \mathrm{V}} \mathrm{h}(v)$.

Let $\mathrm{G}, \mathrm{G}^{\prime}$ be two weighted marked graphs. A (generalized) weighted edge contraction is a map $\pi: X \rightarrow X^{\prime}$ that fulfils the following axioms:

- $\pi$ commutes with commutes with $r, i$, and $h$; 
- the preimage $\pi^{-1}\left(f^{\prime}\right)$ of each half-edge $h^{\prime} \in H\left(G^{\prime}\right)$ consists of precisely one element $f$ (which is necessarily a half-edge of $G$ );

- $\pi$ induces an order preserving bijection $\mathrm{L} \stackrel{\sim}{\longrightarrow} \mathrm{L}^{\prime}$; and

- for every $v^{\prime} \in \mathrm{V}\left(\mathrm{G}^{\prime}\right)$ the preimage $\pi^{-1}(v)$ is a connected finite weighted graph of genus $h\left(v^{\prime}\right)$.

We denote by $\mathrm{J}_{\mathrm{g}, \mathrm{n}}$ the category, whose objects are finite weighted stable graph $\mathrm{G}$ of genus $g$ with $n$ marked legs, and whose morphisms are weighted edge contractions.

\subsection{Tropical curves}

Definition 1.8 Let $\mathrm{P}$ be a monoid. A tropical curve $\Gamma$ over $\mathrm{P}$ is a finite weighted graph $\mathrm{G}(\Gamma)=(\mathrm{G}, \mathrm{h}, \mathrm{m})$ together with a generalized edge length $||:. \mathrm{E}(\mathrm{G}) \rightarrow \mathrm{P}-\{0\}$.

A tropical curve $\Gamma$ (over $\mathrm{P}$ ) is said to be stable, if $\mathrm{G}(\Gamma)$ is stable. The genus $\mathrm{g}(\Gamma)$ of a tropical curve is the genus of the underlying weighted graph $\mathrm{G}(\Gamma)$. A (generalized) weighted edge contraction $\pi: \Gamma \rightarrow \Gamma^{\prime}$ of tropical curves $\Gamma$ over $\mathrm{P}$ and $\Gamma^{\prime}$ over $\mathrm{P}^{\prime}$ consists of a monoid homomorphism $\pi^{b}: P \rightarrow \mathrm{P}^{\prime}$ and a weighted edge contraction $\pi: \mathrm{G}(\Gamma) \rightarrow \mathrm{G}\left(\Gamma^{\prime}\right)$ such that

- $\pi$ contracts an edge if and only $\pi^{b}(|e|)=0$ and

- if $\pi(e)=e \in \mathrm{H}\left(\mathrm{G}^{\prime}\right)$, then $\pi^{b}(|e|)=|\pi(e)|$.

Let $g, n \geqslant 0$ such that $2 g-2+n>0$. By [23, Proposition 2.3], there is a unique stack $\mathcal{M}_{\mathrm{g}, \mathrm{n}}^{\text {trop }}$ over $\left(\mathbf{R P C C}, \tau_{\text {strict }}\right)$, whose fiber over a cone $\sigma$ is the groupoid of stable tropical curves of genus $\mathrm{g}$ with $\mathrm{n}$ marked legs. We refer to $\mathcal{M}_{\mathrm{g}, \mathrm{n}}^{\text {trop }}$ as the moduli stack of tropical curves (of genus g with $\mathrm{n}$ marked points).

Theorem 1.9 ([23] Theorem 1) The stack $\mathcal{M}_{\mathrm{g}, \mathrm{n}}^{\mathrm{trop}}$ is a cone stack.

In fact, one way to prove this, is to realize that $\mathcal{M}_{\mathrm{g}, \mathrm{n}}^{\text {trop }}$ is the cone stack associated to the combinatorial cone stack, defined by the functor

$$
\begin{aligned}
\mathrm{J}_{\mathrm{g}, \mathrm{n}}^{\mathrm{op}} & \longrightarrow \mathbf{R P C}^{f} \\
\mathrm{G} & \longmapsto \sigma_{\mathrm{G}}=\mathbb{R}_{\geqslant 0}^{\mathrm{E}}(\mathrm{G}),
\end{aligned}
$$

where a weighted edge contraction $G \rightarrow G^{\prime}$ naturally induces a face morphism $\sigma_{\mathrm{G}^{\prime}} \rightarrow$ $\sigma_{\mathrm{G}}$.

The moduli stack $\mathcal{M}_{\mathrm{g}, \mathrm{n}}^{\text {trop }}$ does not have faithful monodromy, since there are nontrivial automorphisms of graphs that only induces a trivial permutation of the set of edges. Nevertheless, the image of $\mathrm{J}_{\mathrm{g}, \mathrm{n}}$ in $\mathbf{R P C}^{\mathrm{f}}$ has faithful monodromy and, by [77, Theorem 1.3] the resulting cone stack (with faithful monodromy) functions as a coarse moduli space for $\mathcal{M}_{\mathrm{g}, \mathrm{n}}^{\text {trop }}$, in the sense that it is initial among all strict morphisms $\mathcal{M}_{\mathrm{g}, \mathrm{n}}^{\text {trop }} \rightarrow \mathcal{C}$ to cone stacks with faithful monodromy. In a slight abuse of notation we denote by $M_{\mathrm{g}, \mathfrak{n}}^{\text {trop }}$ both the coarse moduli space of $\mathcal{M}_{\mathrm{g}, \mathfrak{n}}^{\text {trop }}$ and the associated generalized cone complex. 


\section{Uniformization in the tropics}

In this section we first recall from [13,72] the theory of graphs of groups and their fundamental groups. We then use these techniques to expand on [26] and construct tropical Teichmüller space $\mathcal{T}_{\mathrm{g}}^{\mathrm{trop}}$ as a cone stack that is representable by a cone complex $\mathrm{T}_{\mathrm{g}}^{\text {trop }}$.

\subsection{Graphs of groups}

Definition 2.1 A graph of groups $\mathbb{G}$ is a graph $G=(V, E, L)$ together with

- a group $\mathrm{G}_{v}$ for every vertex $v$ of $\mathrm{G}$;

- a group $\mathrm{G}_{f}$ for every half-edge $f$ of $\mathrm{G}$ together with an isomorphism $\mathrm{G}_{f} \stackrel{\sim}{\rightarrow} \mathrm{G}_{i(f)}$ denoted by $\mathrm{g} \mapsto \overline{\mathrm{g}}$; and

- monomorphisms $G_{f} \rightarrow G_{r(f)}$ for every half-edge $f$ of $G$ denoted by $g \mapsto a^{g}$.

The group $G_{v}$ is called the vertex group of the vertex $v \in V(G)$ and $G_{f}$ the edge group of $f \in F(G)$.

A word in a graph of groups $\mathbb{G}$ is a pair $(\gamma, \mathbf{g})$ consisting of a path $\gamma=\left(v_{0}\right.$; $\left.e_{1}, \ldots, e_{n}\right)$ in $G$ consisting of ordered edges $e_{i}=\left[f_{i}<\widetilde{f}_{i}\right]$ connecting $v_{i-1}$ to $v_{i}$ (with $1 \leqslant i \leqslant n)$ and a tuple $\mathbf{g}=\left(g_{0}, \ldots, g_{n}\right)$ of elements $g_{i} \in G_{v_{i}}$. Let $(\gamma, \mathbf{g})$ and $\left(\gamma^{\prime}, \mathbf{g}^{\prime}\right)$ be two words in $\mathbb{G}$ such that the path $\gamma^{\prime}$ starts at the end point of $\gamma$, i.e. for which we have $v_{0}^{\prime}=v_{n+1}$. The concatenation of $(\gamma, \mathbf{g})$ and $\left(\gamma^{\prime}, \mathbf{g}^{\prime}\right)$ is given by the concatenation $\gamma \circ \gamma^{\prime}$ of $\gamma$ and $\gamma^{\prime}$ and the tuple

$$
\mathbf{g} \circ \mathbf{g}^{\prime}=\left(g_{0}, \ldots, g_{n-1}, g_{n} \cdot g_{0}^{\prime}, g_{1}^{\prime}, \ldots, g_{n^{\prime}}^{\prime}\right)
$$

Concatenation of words is associative and, writing $\left(\nu, 1_{\mathrm{G}_{v}}\right)$ for the trival word at the vertex $\nu$, we have $\left(v_{0}, 1_{\mathrm{G}_{v_{0}}}\right) \circ(\gamma, \mathbf{g})=(\gamma, \mathbf{g})=(\gamma, \mathbf{g}) \circ\left(v_{\mathrm{n}}, 1_{\mathrm{G}_{v_{\mathrm{n}}}}\right)$. Moreover, for every word $(\gamma, \mathbf{g})$, there is an inverse word $\left(\gamma^{-1}, \mathbf{g}^{-1}\right)$ given by the inverse $\gamma^{-1}=\left(e_{\mathrm{n}}^{-1}, \ldots, e_{1}^{-1}\right)$ of $\gamma$ and the vector $\mathbf{g}^{-1}=\left(\mathrm{g}_{\mathrm{n}}^{-1}, \ldots, \mathrm{g}_{0}^{-1}\right)$.

Definition 2.2 Let $\mathbb{G}$ be a graph of groups and $v \in V(G)$. The fundamental groupoid $\pi_{1}(\mathbb{G})$ of $\mathbb{G}$ is the groupoid defined as follows:

- Its objects are the vertices of G.

- The set of morphisms between two vertices $v$ and $v^{\prime}$ is the set of words $(\gamma, \mathbf{g})$ in $\mathbb{G}$ modulo the relations generated by

$$
\bar{e}=e^{-1} \text { and } e a^{g} e^{-1}=a^{\bar{g}}
$$

for all oriented edges $e=[f<\widetilde{f}]$ and $g \in G_{f}$. The composition of two morphisms is given by the concatenation of words in $\mathbb{G}$.

For a vertex $v \in V(G)$ the fundamental group $\pi_{1}(\mathbb{G}, v)$ of $\mathbb{G}$ based at $v$ is the group of automorphism of $v$ in $\pi_{1}(\mathbb{G})$. 
Suppose that $G$ is a graph and $\mathbb{G}=(G, 1)$ is the trivial graph of groups on $G$, i.e. the groups $G_{v}$ and $G_{h}$ are all trivial. Then $\pi_{1}(\mathbb{G})=\pi_{1}(G)$, the classical fundamental groupoid of the graph $G$ (defined combinatorially). For every graph of groups $\mathbb{G}$ with underlying graph $G$, the canonical morphism $\mathbb{G} \rightarrow(G, 1)$ induces a surjective homomorphism $\pi_{1}(\mathbb{G}) \rightarrow \pi_{1}(G)$; its kernel is the normal subgroupoid generated by all the $\mathrm{G}_{v}$.

Let $w \in V(G)$ be another base point of $\mathrm{G}$. Let $\gamma$ be a path connecting $v$ to $w$. Then there is a natural isomorphism

$$
\begin{aligned}
\pi_{1}(\mathbb{G}, v) & \stackrel{\sim}{\longrightarrow} \pi_{1}(\mathbb{G}, w) \\
a & \longmapsto \gamma^{-1} \mathrm{a} \gamma .
\end{aligned}
$$

\subsection{From weighted graphs to graphs of groups}

To a weighted graph $(G, h)$ we associate a graph of groups $\mathbb{G}(G, h)$ with underlying graph $G$ by endowing every vertex with the free group $F_{h(v)}$ on $h(v)$ generators and every half-edge $f$ with the trivial group (together with the unique monomorphisms $\left.\mathrm{G}_{\mathrm{f}}=1 \hookrightarrow \mathrm{G}_{\mathrm{r}}(\mathrm{f})\right)$. We refer to $\pi_{1}(\mathbb{G}(\mathrm{G}, \mathrm{h}))$ as well as to $\pi_{1}(\mathbb{G}(\mathrm{G}, \mathrm{h}), v)$ for a base point $\nu \in V(G)$ as the fundamental groupoid of $(G, h)$ and respectively the fundamental group $\pi_{1}(\mathrm{G}, \mathrm{hJ} v)$ of $(\mathrm{G}, \mathrm{h})$ with base point $\left.v\right)$.

Proposition 2.3 For a weighted edge contraction $\phi:\left(\mathrm{G}^{\prime}, \mathrm{h}^{\prime}\right) \rightarrow(\mathrm{G}, \mathrm{h})$ there is an equivalence

$$
\pi_{1}\left(\mathbb{G}\left(G^{\prime}, h^{\prime}\right)\right) \simeq \pi_{1}(\mathbb{G}(G, h))
$$

Proof Let $\phi:\left(\mathrm{G}^{\prime}, \mathrm{h}^{\prime}\right) \rightarrow(\mathrm{G}, \mathrm{h})$ be a weighted edge contraction. Choose base points $v^{\prime} \in V\left(\pi^{-1}(v)\right)$ for every $v \in V\left(G^{\prime}\right)$ and denote by $\mathbb{G}(\pi)$ the graph of groups (with underlying graph $\mathrm{G}$ ) whose group at a vertex $v \in V(G)$ is given by $\pi_{1}\left(\mathbb{G}\left(\pi^{-1}(v)\right) \mathcal{J} v^{\prime}\right)$ (and trivial groups along all edges). The choice of an isomorphism $\pi_{1}\left(\mathbb{G}\left(\pi^{-1}(v)\right) \mathcal{J} v^{\prime}\right) \simeq F_{h(v)}$ induces the desired equivalence $\pi_{1}\left(G^{\prime}, h^{\prime}\right) \simeq$ $\pi_{1}(\mathbb{G}(G, h))$.

Corollary 2.4 For a finite weighted graph $(\mathrm{G}, \mathrm{h})$ of genus $\mathrm{g}$ there is an equivalence

$$
\pi_{1}(\mathbb{G}(G, h)) \simeq F_{g}
$$

Proof Apply Proposition 2.3 to the weighted edge contraction $(\mathrm{G}, \mathrm{h}) \rightarrow(*, \mathrm{~g})$ that contract $G$ to a point with vertex weight $g=g(G, h)$.

This allows us to define the following.

Definition 2.5 Let $(G, h)$ be a finite weighted graph. A Teichmüller marking on ( $G, h)$ is an equivalence

$$
\phi: \pi_{1}(\mathbb{G}(\mathrm{G}, \mathrm{h})) \stackrel{\sim}{\longrightarrow} \mathrm{F}_{\mathrm{g}}
$$


In other words, a Teichmüller marking is an outer isomorphism class $\phi_{v}: \pi_{1}(\mathbb{G}, v) \stackrel{\sim}{\longrightarrow}$ $F_{g}$ for one (and, since $G$ is connected, all) $v \in V(G)$.

Definition 2.6 Let $(G, h)$ be a finite weighted graph of genus g. Two equivalences $\phi_{i}: \pi_{1}(G, h) \stackrel{\sim}{\rightarrow} F_{g}$ (for $i=1,2$ ) are said to be topologically equivalent, if for one (and therefore all) $v \in \mathrm{V}(\mathrm{G})$ the induced surjective homomorphisms

$$
\mathrm{F}_{\mathrm{g}} \stackrel{\phi_{\mathrm{i}}}{\longrightarrow} \pi_{1}(\mathbb{G}(\mathrm{G}, \mathrm{h}), v) \longrightarrow \pi_{1}(\mathrm{G}, v)
$$

for $i=1,2$ are equal.

Topological equivalence defines an equivalence relation on the class of all Teichmüller markings; we write $\left[\phi: \mathbb{G}(\Gamma) \stackrel{\sim}{\rightarrow} \mathrm{F}_{\mathrm{g}}\right]$ for the topological equivalence class associated to a Teichmüller marking.

\subsection{Tropical Teichmüller space}

Given a tropical curve $\Gamma$ (over a monoid P), we write $\mathbb{G}(\Gamma)$ for the graph of groups associated to the underlying weighted graph $(\mathrm{G}, \mathrm{h})$ of $\Gamma$. Moreover, we denote $\pi_{1}(\Gamma)=$ $\pi_{1}(\mathbb{G}(\Gamma))$ and $\pi_{1}(\Gamma, v)=\pi_{1}(\mathbb{G}(\Gamma), v)$ for $v \in \mathrm{V}(\mathrm{G})$. A Teichmüller marking on a tropical curve $\Gamma$ is a Teichmüller marking on the underlying finite weighted graph $\mathrm{G}(\Gamma)$.

Let $\mathrm{g} \geqslant 2$. By [23, Proposition 2.3] there is a unique stack $\mathcal{T}_{\mathrm{g}}^{\text {trop }}$ over (RPCC, $\left.\tau_{\text {strict }}\right)$ whose fiber over a rational polyhedral cone $\sigma$ is the groupoid of pairs consisting of a stable tropical curve $\Gamma$ of genus $g$ together with a topological equivalence class of Teichmüller marking $\left[\phi: \mathbb{G}(\Gamma) \stackrel{\sim}{\rightarrow} \mathrm{F}_{\mathrm{g}}\right]$.

Theorem 2.7 The space $\mathcal{T}_{\mathrm{g}}^{\mathrm{trop}}$ is representable by a cone complex $\mathrm{T}_{\mathrm{g}}^{\mathrm{trop}}$.

Following [26] we introduce the following terminology.

Definition 2.8 The cone complex $\mathrm{T}_{\mathrm{g}}^{\mathrm{trop}}$ is called tropical Teichmüller space.

Proof of Theorem 2.7 Consider the category $\widetilde{J}_{g}$ whose objects are tuples $(G, h,[\phi])$ consisting of a vertex-weighted graph $(G, h)$ of genus $g$ and a topological equivalence class of a Teichmüller marking $\left[\phi: \mathbb{G}(\mathrm{G}, \mathrm{h}) \stackrel{\sim}{\longrightarrow} \mathrm{F}_{\mathrm{g}}\right]$ and whose morphism are weighted edge contractions. We note that, for a weighted edge contraction $(G, h) \rightarrow$ $\left(G^{\prime}, h^{\prime}\right)$, a Teichmüller marking $\phi: \pi_{1}(\mathbb{G}(G, h)) \stackrel{\sim}{\rightarrow} F_{g}$ naturally induces a Teichmüller marking of $\left(\mathrm{G}^{\prime}, \mathrm{h}^{\prime}\right)$ by Proposition 2.3. The natural function $\widetilde{\mathrm{J}}_{\mathrm{g}} \rightarrow$ RPC $^{f}$ given by $(\mathrm{G}, \mathrm{h},[\phi]) \mapsto \sigma_{(\mathrm{G}, \mathrm{h},[\phi])}=\mathbb{R}_{\geqslant 0}^{\mathrm{E}(\mathrm{G})}$ makes $\widetilde{J}_{\mathrm{g}}$ into a category fibered in groupoids over RPC ${ }^{f}$, i.e. into a combinatorial cone stack. The associated cone stack is equivalent to $\mathcal{T}_{\mathrm{g}}^{\text {trop }}$, since strict morphisms $\sigma \rightarrow \mathcal{T}_{\mathrm{g}}^{\text {trop }}$ from rational polyhedral cones naturally correspond to objects in $\widetilde{\mathrm{J}}_{\mathrm{g}}$.

We now show that $\widetilde{J}_{g} \rightarrow$ RPC ${ }^{f}$ defines a cone complex: The operation of the automorphism group of a finite graph on its fundamental groupoid is faithful. Therefore 
the automorphism group of $(\mathrm{G}, \mathrm{h},[\phi])$ in $\widetilde{\mathrm{J}}_{\mathrm{g}}$ is trivial and thus $\widetilde{\mathrm{J}}_{\mathrm{g}} \rightarrow \mathbf{R P C} \mathbf{C}^{\mathrm{f}}$ is fibered in sets (and not groupoids). It is a poset, since, whenever we have two weighted edge contraction $(G, h) \rightrightarrows\left(G^{\prime}, h^{\prime}\right)$, there already is an automorphism of $(G, h)$ that makes the diagram

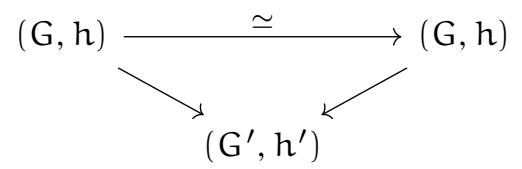

commute. Thus the colimit of the diagram $\widetilde{J}_{g} \rightarrow \mathbf{R P C}{ }^{f}$ is a cone complex $T_{g}^{\text {trop }}$.

Remark 2.9 The locus of pairs $(\Gamma,[\phi])$ in $\mathrm{T}_{\mathrm{g}}^{\text {trop }}$ where the vertex weight function is trivial is precisely the equal to space of metric graphs together with a Teichmüller marking $\phi: \pi_{1}(\Gamma) \stackrel{\sim}{\rightarrow} F_{g}$ (without reference to topological equivalence, since all vertex groups of $\mathbb{G}(\Gamma)$ are trivial). As explained in [26, Section 3.2] this space is naturally homeomorphic to the (not volume-normalized) Outer space in the sense of Culler and Vogtmann [28]. In [28] the authors impose the that for metric graphs $\Gamma$ in $\mathrm{CV}_{\mathrm{g}}$ the condition

$$
\sum_{e \in E(\Gamma)}|e|=1
$$

on the total length of $\Gamma$ holds. As in [26], we do not follow this convention.

\subsection{The quotient by Out $\left(F_{g}\right)$}

There is natural operation of the group $\operatorname{Aut}\left(\mathrm{F}_{\mathrm{g}}\right)$ on $\mathcal{T}_{\mathrm{g}}^{\text {trop }}$ that is given by sending $(\Gamma,[\phi])$ to $(\Gamma,[\mathrm{g} \circ \phi])$ for $g \in \operatorname{Aut}\left(F_{g}\right)$. An equivalence $\phi: \pi_{1}(\Gamma) \stackrel{\sim}{\rightarrow} F_{g}$ is determined only up to inner automorphisms of $F_{g}$ and so the group $\operatorname{Inn}\left(F_{g}\right)$ of inner automorphisms of $\mathrm{F}_{\mathrm{g}}$ acts trivially on $\mathcal{T}_{\mathrm{g}}^{\text {trop }}$. Thus there is a natural induced operation of $\operatorname{Out}\left(\mathrm{F}_{\mathrm{g}}\right)=\operatorname{Aut}\left(\mathrm{F}_{\mathrm{g}}\right) / \operatorname{Inn}\left(\mathrm{F}_{\mathrm{g}}\right)$ on $\mathcal{T}_{\mathrm{g}}^{\text {trop }}$.

Consider now the natural morphism $\mathcal{T}_{\mathrm{g}}^{\text {trop }} \rightarrow \mathcal{M}_{\mathrm{g}}^{\text {trop }}$ that is given by forgetting the Teichmüller marking. Since $\operatorname{Out}\left(\mathrm{F}_{\mathrm{g}}\right)$ only operates on the markings, the map $\mathcal{T}_{\mathrm{g}}^{\text {trop }} \rightarrow \mathcal{M}_{\mathrm{g}}^{\text {trop }}$ is invariant under this operation and there is an induced morphism $\left[\mathcal{T}_{\mathrm{g}}^{\text {trop }} / \operatorname{Out}\left(\mathrm{F}_{\mathrm{g}}\right)\right] \rightarrow \mathcal{M}_{\mathrm{g}}^{\text {trop }}$.

Theorem 2.10 The relative coarse moduli space of $\left[\mathcal{T}_{\mathrm{g}}^{\text {trop }} / \operatorname{Out}\left(\mathrm{F}_{\mathrm{g}}\right)\right]$ over $\mathcal{M}_{\mathrm{g}}^{\text {trop }}$ is naturally equivalent to $\mathcal{M}_{\mathrm{g}}^{\text {trop }}$.

Proof The induced morphism $\left[\mathcal{T}_{\mathrm{g}}^{\text {trop }} / \operatorname{Out}\left(\mathrm{F}_{\mathrm{g}}\right)\right] \rightarrow \mathcal{M}_{\mathrm{g}}^{\text {trop }}$ is essentially surjective, since every tropical curve $\Gamma$ can be endowed with a Teichmüller marking $\phi: \pi_{1}(\Gamma) \stackrel{\sim}{\longrightarrow}$ $\mathrm{F}_{\mathrm{g}}$. It is full, since every weighted edge contraction $\phi: \Gamma \rightarrow \Gamma^{\prime}$ induces an equivalence $\pi_{1}(\Gamma) \stackrel{\sim}{\rightarrow} \pi_{1}\left(\Gamma^{\prime}\right)$ by Proposition 2.3. Going from $\left[\mathcal{T}_{\mathrm{g}}^{\mathrm{trop}} / \operatorname{Out}\left(\mathrm{F}_{\mathrm{g}}\right)\right]$ to the relative 
coarse moduli space over $\mathcal{M}_{\mathrm{g}}^{\text {trop }}$ makes the induced map also faithful and thus the result follows.

\section{Lifting via Artin fans}

In this section we use methods from logarithmic geometry in the sense of KatoFontaine-Illusie [44] and, in particular, the theory of Artin fans (as in [5,6,12,23,77]), to lift tropical Teichmüller space to the world of algebraic geometry and to study the process of tropicalization.

\subsection{Logarithmic structures}

Recall from [44] that a logarithmic structure on a scheme $\underline{X}$ is a pair $\left(M_{X}, \alpha_{X}\right)$ consisting of

- a sheaf of monoids $M_{X}$ defined on the étale topology on $\underline{X}$, and

- a monoid homomorphism $\alpha_{X}: M_{X} \rightarrow\left(\mathcal{O}_{X}, \cdot\right)$ that induces an isomorphism $\alpha_{\bar{X}}^{-1} \mathcal{O}_{\underline{X}}^{*} \simeq \mathcal{O}_{\underline{X}}^{*}$

We refer to the tuple $X=\left(\underline{X}, M_{X}, \alpha_{X}\right)$ consisting of a scheme $\underline{X}$ and a logarithmic structure $\left(M_{X}, \alpha_{X}\right)$ as a logarithmic scheme. Whenever convenient we drop the reference to $\alpha_{X}$ and simply write $X=\left(\underline{X}, \alpha_{X}\right)$ for a logarithmic scheme. We write $\bar{M}_{X}$ for the quotient $\bar{M}_{X}=M_{X} / M_{X}^{*}$, which is known as the characteristic monoid of $X$.

A logarithmic scheme $X$ is called fine and saturated if étale locally there is a homomorphism $\mathrm{P}_{\underline{X}} \rightarrow\left(\mathcal{O}_{\underline{X}}, \cdot\right)$ from the constant sheaf $\mathrm{P}_{\underline{X}}$ associated to a fine and saturated monoid $\overline{\mathrm{P}}$ to $\left(\mathcal{O}_{\underline{X}}, \cdot\right)$ such that the logarithmic structure $M_{X}$ is given via the pushout square

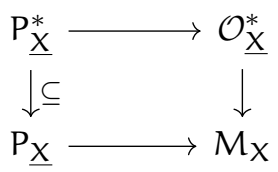

For further details on logarithmic geometry we refer the avid reader to [44], [2], and [60]. From now on the terms logarithmic scheme or logarithmic stack will always refer to a fine and saturated logarithmic scheme or logarithmic stack. We denote the category of (fine and saturated) logarithmic scheme by LSch and the category of (fine and saturated) logarithmic stacks by LSt.

\subsection{Logarithmic curves}

A logarithmic curve over a logarithmic base scheme $S$ is a logarithmically smooth morphism $X \rightarrow S$ that is proper, integral, saturated, and has geometrically connected fibers of dimension one. 
Theorem 3.1 ([46] Theorem 1.3) Let $\mathrm{X} \rightarrow \mathrm{S}$ be a logarithmic curve. Then, étale locally on $\mathrm{S}$, every point $\mathrm{x}$ of $\mathrm{X}$ has an étale neighborhood $\mathrm{V}$ together with a morphism $\pi: \mathrm{V} \rightarrow \mathrm{S}$ such that one of the following holds:

(i) $\mathrm{V}=\operatorname{Spec} \mathcal{O}_{\mathrm{S}}[\mathrm{u}]$ with $\mathrm{M}_{\mathrm{V}}=\pi^{*} \mathrm{M}_{\mathrm{S}}$;

(ii) $\mathrm{V}=\operatorname{Spec} \mathcal{O}_{\mathrm{S}}[\mathrm{u}]$ with $\mathrm{M}_{\mathrm{V}}=\pi^{*} \mathrm{M}_{\mathrm{S}} \oplus \mathbb{N} v$ with $\alpha_{\mathrm{V}}(v)=u$; or

(iii) $\mathrm{V}=\operatorname{Spec} \mathcal{O}_{S}[x, y] /(x y-t)$ for some $t \in \mathcal{O}_{S}$ and

$$
M_{\mathrm{V}}=\pi^{*} M_{S} \oplus \mathbb{N} \alpha \oplus \mathbb{N} \beta /(\alpha+\beta=\delta)
$$

for some $\delta \in \pi^{*} M_{S}$ with $\epsilon_{V}(\alpha)=x, \epsilon_{V}(\beta)=y$, and $\epsilon_{S}(\delta)=t$.

So the underlying family of curves $\bar{X} \rightarrow \bar{S}$ is flat and proper, and each fiber is a nodal curve with a finite number of (a priori unordered) sections that do not meet the singularities in each fiber. We define the moduli stack $\mathcal{M}_{\mathrm{g}, \mathrm{n}}^{\log }$ of logarithmic curves to be the unique stack over LSch whose fiber over a logarithmic base scheme $S$ is the groupoid of stable logarithmic curves of genus $g$ with $n$ marked sections.

The connection with the classical Deligne-Knudsen-Mumford moduli stack $\overline{\mathcal{M}}_{\mathrm{g}, \mathrm{n}}$ is established by the following:

Theorem 3.2 ([46] Theorem 4.5) The moduli stack is represented by the pair $\left(\overline{\mathcal{M}}_{\mathrm{g}, \mathrm{n}}, \mathrm{M}_{\mathrm{g}, \mathrm{n}}\right)$ where $\mathrm{M}_{\overline{\mathcal{M}}_{\mathrm{g}, \mathrm{n}}}$ is the divisorial logarithmic structure associated to the boundary divisor of $\overline{\mathcal{M}}_{\mathrm{g}, \mathrm{n}}$, i.e. the complement of the locus $\mathcal{M}_{\mathrm{g}, \mathrm{n}}$ of smooth $\mathrm{n}$-marked curves of genus $\mathrm{g}$ in $\overline{\mathcal{M}}_{\mathrm{g}, \mathrm{n}}$.

\subsection{From cone stacks to Artin fans}

Let $\sigma$ be a rational polyhedral cone. Denote by $\mathcal{A}_{\sigma}$ the quotient stack

$$
\mathcal{A}_{\sigma}=\left[\operatorname{Spec} \mathbb{Z}\left[S_{\sigma}\right] / \operatorname{Spec} \mathbb{Z}\left[S_{\sigma}^{g p}\right]\right]
$$

of the affine toric variety Spec $\mathbb{Z}\left[S_{\sigma}\right]$ by the diagonalizable group $\operatorname{Spec} \mathbb{Z}\left[S_{\sigma}^{g p}\right]$. By [61, Proposition 5.17], for every logarithmic scheme $X$ there is a natural isomorphism

$$
\operatorname{Hom}_{\mathbf{L S t}}\left(\mathrm{X}, \mathcal{A}_{\sigma}\right)=\operatorname{Hom}_{\mathbf{M o n}}\left(\mathrm{S}_{\sigma}, \overline{\mathrm{M}}_{\mathrm{X}}\right)
$$

As in [77] this observation implies that the association $\sigma \mapsto \mathcal{A}_{\sigma}$ defines full and faithful functor from RPC to the category of logarithmic stacks. We refer to a logarithmic stack of the form $\mathcal{A}_{\sigma}$ as an Artin cone.

Definition 3.3 An Artin fan is a logarithmic algebraic stack that admits a cover by a disjoint union of Artin cones that is strict and étale.

In [23] we have seen the following:

Theorem 3.4 ([23] Theorem 3) The category of Artin fans is naturally equivalent to the category of cone stacks. 
When $\tau$ is a face of a rational polyhedral cone $\sigma$, the induced homomorphism $\mathrm{S}_{\sigma} \rightarrow \mathrm{S}_{\tau}$ determines an open immersion $\mathcal{A}_{\tau} \subseteq \mathcal{A}_{\sigma}$. So, if $\Sigma$ is a rational polyhedral cone complex, then we may construct the associated Artin fan $\mathcal{A}_{\Sigma}$ as

$$
\mathcal{A}_{\Sigma}=\bigcup_{\sigma \subseteq \Sigma} \mathcal{A}_{\sigma} .
$$

In general, given a cone $\operatorname{stack} \mathcal{C}$, we may choose a strict groupoid presentation $[\mathrm{U} / \mathrm{R}] \simeq$ $\mathcal{C}$ in RPCC and construct the associated Artin fan $\mathcal{A}_{\mathcal{C}}$ as the quotient of the induced strict étale groupoid

$$
\mathcal{A}_{\mathrm{R}} \rightrightarrows \mathcal{A}_{\mathrm{U}}
$$

Remark 3.5 In [23] the proof of Theorem 3.4 is only written for logarithmic schemes over a field $k$. It, however, directly generalizes to logarithmic schemes over $\mathbb{Z}$ (and in fact to any other logarithmic base scheme with trivial logarithmic structure).

In order to keep our notation less bulky, we usually denote both the cone stack $\mathcal{C}$ and the associated Artin fan $\mathcal{A}_{\mathcal{C}}$ with the same letter $\mathcal{C}$.

\subsection{Construction of $\mathcal{T}_{\mathrm{g}}^{\mathrm{log}}$}

Let $g, n \geqslant 0$ such that $2 g-2+n>0$. By [23, Theorem 4] the Artin fan associated to the tropical moduli stack $\mathcal{M}_{\mathrm{g}, \mathrm{n}}^{\text {trop }}$ is the category whose fiber over a logarithmic scheme $S$ is the groupoid of families of tropical curves over S. A family of tropical curves over $\mathrm{S}$ consists of

- a collection $\left(\Gamma_{\mathrm{s}}\right)$ of tropical curves $\Gamma_{\mathrm{s}} \in \mathcal{M}_{\mathrm{g}, \mathrm{n}}^{\mathrm{trop}}\left(\overline{\mathrm{M}}_{\mathrm{S}}\right)$ with edge lengths in the characteristic monoid $\bar{M}_{S}$ indexed by all geometric points $s$ of $S$; and

- for every étale specialization $t \rightsquigarrow s$ of geometric points of $S$ a weighted edge contraction $\Gamma_{\mathrm{s}} \rightarrow \Gamma_{\mathrm{t}}$ such that, whenever $\Gamma_{\mathrm{s}}$ is metrized via the composition |. $\left.\right|_{t \rightsquigarrow s}: E(\Gamma) \rightarrow \bar{M}_{S, s} \rightarrow \bar{M}_{S, t}$, the tropical curve $\Gamma_{t}$ is given by contracting those edges $e$ in $\Gamma_{\mathrm{s}}$ for which $|e|_{t \rightsquigarrow s}=0$.

Again by [23, Theorem 4], there is a natural modular logarithmic tropicalization morphism

$$
\operatorname{trop}_{\mathrm{g}, \mathrm{n}}^{\log }: \mathcal{M}_{\mathrm{g}, \mathrm{n}}^{\log } \longrightarrow \mathcal{M}_{\mathrm{g}, \mathrm{n}}^{\mathrm{trop}}
$$

that is strict, smooth, and surjective. It is given by associating to a logarithmic curve $X \rightarrow S$ the family $\left(\Gamma_{X_{s}}\right)$ of dual tropical curves of each fiber $X_{S}$ over a geometric point $s$ of $S$. The dual tropical curve $\Gamma_{X}$ of a logarithmic curve $X$ over a logarithmic point $S$ is defined as follows:

- the underlying graph $\mathrm{G}_{X}$ is the dual graph of the stable curve $\underline{X}$, so that its vertices $v$ correspond to the irreducible components $X_{v}$ of $\underline{X}$, an edge connecting two vertices $v, v^{\prime}$ to a node connecting the two components $X_{v}$ and $X_{v^{\prime}}$ and the legs of $G_{X}$ emanating from $v$ correspond to the marked points on $X_{v}$; 
- the vertex weight $h(v)$ is the genus of the normalization $\widetilde{X}_{v}$ of $X_{v}$; and

- the edge length $|e| \in \bar{M}_{S, s}$ of an edge $e$ of $G(X)$ is the logarithmic deformation parameter $\delta_{e} \in \bar{M}_{S, s}$ at the node $p_{e}$, as explained in Theorem 3.1 (ii) above.

Definition 3.6 We define $\mathcal{T}_{\mathrm{g}}^{\mathrm{log}}$ to be the fiber product

$$
\mathcal{T}_{\mathrm{g}}^{\text {log }}=\mathcal{M}_{\mathrm{g}}^{\text {log }} \times{ }_{\mathcal{M}_{\mathrm{g}}^{\text {trop }}} \mathcal{T}_{\mathrm{g}}^{\text {trop }}
$$

over the logarithmic tropicalization morphism trop ${ }_{\mathrm{g}}^{\log }: \mathcal{M}_{\mathrm{g}}^{\mathrm{log}} \rightarrow \mathcal{M}_{\mathrm{g}}^{\text {trop }}$ and the natural morphism $\mathcal{T}_{\mathrm{g}}^{\mathrm{trop}} \rightarrow \mathcal{M}_{\mathrm{g}}^{\text {trop }}$ that forgets the Teichmüller marking.

Using the above description of $\mathcal{M}_{\mathrm{g}}^{\text {trop }}$ as a stack over LSch the stack $\mathcal{T}_{\mathrm{g}}^{\text {tog }}$ is the category whose fiber over a logarithmic scheme $S$ is the groupoid of logarithmic curves $X \rightarrow S$ in $\mathcal{M}_{\mathrm{g}}^{\log }(\mathrm{S})$ together with a topological equivalence class of a Teichmüller marking $\left[\phi_{s}: \pi_{1}\left(\Gamma_{s}\right) \stackrel{\sim}{\rightarrow} F_{g}\right]$ on every dual tropical curve $\Gamma_{s}$ (where $s$ are the geometric points of $S$ ) that are compatible with étale specialization.

Theorem 3.7 The logarithmic stack $\mathcal{T}_{\mathrm{g}}^{\mathrm{log}}$ is representable by a pair $\left(\underline{\mathcal{I}}_{\mathrm{g}}^{\mathrm{log}}, M_{\mathcal{S}_{\mathrm{g}, \mathrm{n}}^{\mathrm{log}}}\right)$ consisting of:

(i) a Deligne-Mumford stack $\underline{\mathcal{T}}_{\mathrm{g}}^{\mathrm{log}}$, that is smooth, universally closed, and locally of finite type over $\mathbb{Z}$; and

(ii) a fine and saturated logarithmic structure $M_{\mathcal{T}_{\mathrm{g}}^{\mathrm{log}}}$ that is associated to the complement of the locus of smooth curves in $\underline{\mathcal{T}}_{\mathrm{g}}^{\mathrm{log}}$, which has normal crossings over $\mathbb{Z}$.

Proof We may define $\underline{\mathcal{I}}_{\mathrm{g}}^{\log }$ as the fibered product

$$
\underline{\mathcal{T}}_{\mathrm{g}}^{\text {tog }}=\overline{\mathcal{M}}_{\mathrm{g}} \times{\underline{\mathcal{M}_{\mathrm{g}}^{\text {trop }}}}_{\underline{\mathcal{T}}_{\mathrm{g}}^{\text {trop }}} .
$$

Since $\mathcal{T}_{\mathrm{g}}^{\text {trop }} \rightarrow \mathcal{M}_{\mathrm{g}}^{\text {trop }}$ is strict and surjective, it immediately follows that $\underline{\mathcal{I}}_{\mathrm{g}}^{\text {log }}$ is smooth, universally closed, and locally of finite type over $\mathbb{Z}$. We endow $\underline{\mathcal{I}}_{\mathrm{g}}^{\text {log }}$ with the logarithmic structure $M_{\mathcal{T}_{\mathrm{g}}^{\text {log }}}$ that is associated to the pullback of the boundary divisor of $\overline{\mathcal{M}}_{\mathrm{g}}$, which has normal crossings, since the boundary divisor on $\overline{\mathcal{M}}_{\mathrm{g}}$ has normal crossings on the map $\mathcal{T}_{\mathrm{g}}^{\text {trop }} \rightarrow \mathcal{M}_{\mathrm{g}}^{\text {trop }}$ is strict and therefore also smooth (in fact, étale locally an isomorphism). By [23, Theorem 4], the tropicalization morphism $\mathcal{M}_{\mathrm{g}}^{\text {log }} \rightarrow \mathcal{M}_{\mathrm{g}}^{\text {trop }}$ is strict and therefore we have

$$
\mathcal{T}_{\mathrm{g}}^{\text {log }} \simeq \mathcal{M}_{\mathrm{g}}^{\log } \times_{\underline{\mathcal{M}}_{\mathrm{g}}^{\text {trop }}} \underline{\mathcal{T}}_{\mathrm{g}}^{\text {trop }}
$$

This, together with [46, Theorem 4.5], i.e. Theorem 3.2 above, implies our claim.

Remark 3.8 The moduli stack $\mathcal{T}_{\mathrm{g}}^{\mathrm{log}}$ is not separated. The reason is that e.g. in a stable degeneration of a smooth curve, we have exactly one equivalence class of Teichmüller 
markings in the generic fiber and very many in the special fiber, whenever its dual graph has non-trivial cycles.

Remark 3.9 It follows a posteriori from Theorem 3.14 that the boundary divisor of $\underline{\mathcal{S}}_{\mathrm{g}}$ even has simple normal crossings, i.e. that all strata of the boundary divisor are smooth over $\mathbb{Z}$.

The following Theorem 3.10 lifts Theorem 2.10 to the logarithmic category.

Theorem 3.10 The relative coarse moduli space of the stack quotient $\left[\mathcal{T}_{\mathrm{g}}^{\mathrm{log}} / \operatorname{Out}\left(\mathrm{F}_{\mathrm{g}}\right)\right]$ over $\mathcal{M}_{\mathrm{g}}^{\log }$ is equivalent to $\overline{\mathcal{M}}_{\mathrm{g}}^{\log }$.

Proof By Theorem 2.10 the relative coarse moduli space of the stack quotient $\left[\mathcal{T}_{\mathrm{g}}^{\text {trop }} / \operatorname{Out}\left(\mathrm{F}_{\mathrm{g}}\right)\right]$ over $\mathcal{M}_{\mathrm{g}}^{\text {trop }}$ is equivalent to $\mathcal{M}_{\mathrm{g}}^{\text {trop }}$. The claim is an immediate consequence of this and of the definition of $\mathcal{T}_{\mathrm{g}}^{\text {log }}$ as a fibered product $\mathcal{M}_{\mathrm{g}}^{\text {log }} \times \mathcal{M}_{\mathrm{g}}^{\text {trop }}$ $\mathcal{T}_{\mathrm{g}}^{\text {trop }}$

\subsection{From the fundamental category of a logarithmic stack to its tropicalization}

Let $\mathcal{X}$ be a logarithmic algebraic stack. Denote by $\widetilde{\Pi}_{1}(\mathcal{X})$ the category whose objects are the geometric points $\chi \rightarrow \mathcal{X}$ and whose morphisms are étale specializations $x \rightsquigarrow y$ in $\mathcal{X}$. We say that an étale specialization $x \rightsquigarrow y$ is strict, if the induced map $\bar{M}_{\mathcal{X}, y} \rightarrow$ $\bar{M}_{\mathcal{X}, \chi}$ is an isomorphism.

Definition 3.11 The fundamental category $\Pi_{1}(\mathcal{X})$ of a logarithmic stack $\mathcal{X}$ is defined to be the localization of $\widetilde{\Pi}_{1}(\mathcal{X})$ along the class of strict specializations.

For a geometric point $x \rightarrow \mathcal{X}$ we write $\sigma_{x}$ for the rational polyhedral cone Hom $\left(\bar{M}_{\mathcal{X}, x}, \mathbb{R}_{\geqslant 0}\right)$. For an étale specialization $x \rightsquigarrow y$ the induced morphism $\sigma_{x} \rightarrow \sigma_{y}$ is a face morphism and, whenever $x \rightsquigarrow y$ is strict, this map is an isomorphism. So there is a natural functor $\Pi_{1}(\mathcal{X}) \rightarrow$ RPC $^{f}$ given by the association $x \mapsto \sigma_{x}$.

Proposition 3.12 If $\mathcal{X}$ is a logarithmically smooth (over a base scheme $\mathrm{S}$ with trivial logarithmic structure), then the functor $\chi \mapsto \sigma_{\chi}$ makes $\Pi_{1}(\mathcal{X})$ into a category fibered in groupoids over $\boldsymbol{R P C}$.

Proof We may check the two axioms of a category fibered in groupoids étale locally on $\mathcal{X}$ and so we may assume that $\mathcal{X}$ is an affine toric variety $\operatorname{Spec} \mathcal{O}_{\mathrm{S}}[\mathrm{P}]$ over $\mathrm{S}$ (where $\mathrm{P}$ is a fine and saturated monoid). In this case $\Pi_{1}\left(\operatorname{Spec} \mathcal{O}_{S}[\mathrm{P}]\right)$ is equivalent to the poset of (generic points of) torus orbits of $\operatorname{Spec} \mathcal{O}_{S}[\mathrm{P}]$. Then the statement follows from the order-reversing correspondence between torus orbits of $\operatorname{Spec} \mathcal{O}_{S}[\mathrm{P}]$ and faces of $\sigma_{P}=\operatorname{Hom}\left(P, \mathbb{R}_{\geqslant 0}\right)$.

In other words, Proposition 3.12 tells us that $\Pi_{1}(\mathcal{X})$ is a combinatorial cone stack. As explained in Proposition 1.6 above, we may associate to $\Pi_{1}(\mathcal{X})$ its coarse moduli 


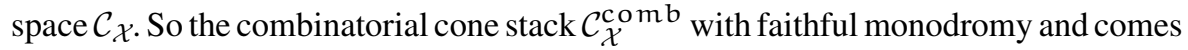
with a strict morphism $\Pi_{1}(\mathcal{X}) \rightarrow \mathcal{C}_{\mathcal{X}}$ that is initial among all strict morphisms to cone stacks with faithful monodromy.

Proposition 3.13 Let $\mathcal{X}$ be logarithmically smooth (over a base scheme $\mathrm{S}$ with trivial logarithmic structure). Then there is a strict morphism $\mathcal{X} \rightarrow \mathcal{C}_{\mathcal{X}}$ that is initial among strict morphism to cone stacks with faithful monodromy.

Here, we again lift $\mathcal{C}_{\mathcal{X}}$ to the category of logarithmic stacks as an Artin fan. In [6, Propositon 3.1.1] we see that every (reasonable) logarithmic stack $\mathcal{X}$ admits a strict morphism $\mathcal{X} \rightarrow \mathcal{A}_{\mathcal{X}}$ to an Artin fan with faithful monodromy that is initial among all strict morphisms to Artin fans with faithful monodromy. When $\mathcal{X}$ is logarithmically smooth, Proposition 3.13 tells us that the Artin fan associated to $\mathcal{C}_{\mathcal{X}}$ is equivalent to $\mathcal{A}_{\mathcal{X}}$ so that the diagram

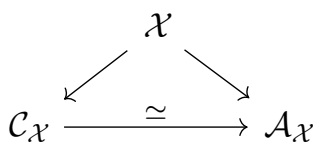

commutes. We refer to trop ${ }_{\mathcal{X}}: \mathrm{X} \rightarrow \mathcal{C}_{\mathcal{X}}$ as the logarithmic tropicalization morphism associated to $\mathcal{X}$ and to $\mathcal{C}_{\mathcal{X}}$ as the logarithmic tropicalization of $\mathcal{X}$.

Proof of Proposition 3.13 Let us first assume that $\mathcal{X}$ is represented by a logarithmically smooth scheme $X$ that is small, i.e. that $X$ has a unique closed logarithmic stratum. In this case, the lift of $\mathcal{C}_{X}$ is given by $\mathcal{A}_{\sigma}$ where $\sigma$ is the rational polyhedral cone $\operatorname{Hom}\left(\bar{M}_{X}, \mathbb{R}_{\geqslant 0}\right)$ dual to the characteristic monoid of $X$. The strict morphism $X \rightarrow \mathcal{A}_{\sigma}$ is the one associated to the identity under the natural correspondence

$$
\operatorname{Hom}_{\mathbf{L S t}}\left(\mathrm{X}, \mathcal{A}_{\sigma}\right)=\operatorname{Hom}_{\mathbf{M o n}}\left(\mathrm{S}_{\sigma}, \overline{\mathrm{M}}_{\mathrm{X}}\right)
$$

from [61, Proposition 5.17]. We may now continue our argument as in [6, Proposition 3.1.1] and show that this morphism is initial among strict morphisms to Artin fans with faithful monodromy. In the general situation (when $\mathcal{X}$ is not small), both $\mathcal{A}_{\mathcal{X}}$ and $\mathcal{C}_{\mathcal{X}}$ arises as colimits of representable morphisms over Olsson's stack LOGS of logarithmic structures over $S$ (as introduced in [61]) and therefore both constructions agree.

\subsection{Tropicalization of $\mathcal{M}_{\mathrm{g}}^{\log }$ and $\mathcal{T}_{\mathrm{g}}^{\mathrm{log}}$}

The following Theorem 3.14 will imply Theorem B from the introduction (see Theorem 4.4 below). In the case of $\mathcal{M}_{\mathrm{g}}^{\text {log }}$ it also rephrases [77, Theorem 1.3].

Theorem 3.14 The logarithmic tropicalization of $\mathcal{M}_{\mathrm{g}}^{\mathrm{log}}$ is isomorphic to the coarse moduli space $\mathrm{M}_{\mathrm{g}}^{\text {trop }}$ and the logarithmic tropicalization of $\mathcal{T}_{\mathrm{g}}^{\mathrm{log}}$ is equivalent to 
$\mathcal{T}_{\mathrm{g}}^{\text {trop }}$ so that the natural diagram

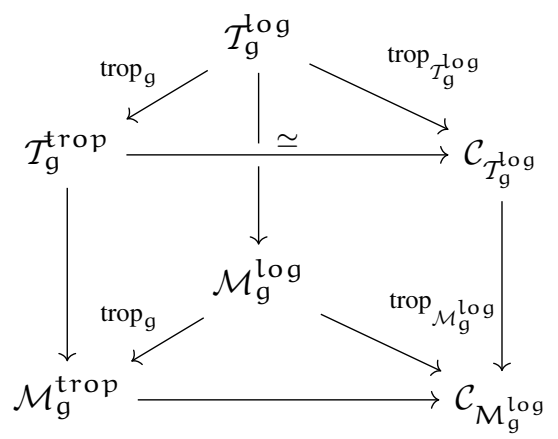

commutes.

Proof The first step of the proof consists of a stack-theoretic and logarithmic reinterpretation of the proof of the main result in [7]. It is based on the following facts, which be found e.g. in [1] and [7]:

- The boundary strata of $\overline{\mathcal{M}}_{\mathrm{g}}$ are in natural one-to-one correspondence with stable weighted graphs of genus $\mathrm{g}$.

- An étale specialization $\eta_{\mathrm{G}} \rightsquigarrow \eta_{\mathrm{G}^{\prime}}$ of generic points of boundary strata that is not an isomorphism corresponds to a weighted edge contraction $\mathrm{G} \rightarrow \mathrm{G}^{\prime}$.

- There is an isomorphism $\bar{M}_{\eta_{\mathrm{G}}} \simeq \mathbb{N}^{\mathrm{E}(\mathrm{G})}$ such that the group of automorphisms of $\bar{M}_{\eta_{\mathrm{G}}}$ induced by self-specializations $\eta_{\mathrm{G}} \stackrel{\sim}{\rightarrow} \eta_{\mathrm{G}}$ (called the monodromy group of the stratum in [7]) agrees with the group of permutations of $\mathbb{N} E(G)$ induced by automorphisms of $\mathrm{G}$.

These three facts together imply that there is a natural equivalence between the coarse moduli space of $\mathcal{M}_{\mathrm{g}}^{\text {trop }}$ and $\mathcal{C}_{\mathcal{M}_{\mathrm{g}}^{\text {log }}}$. Since, by [23, Theorem 4] the tropicalization

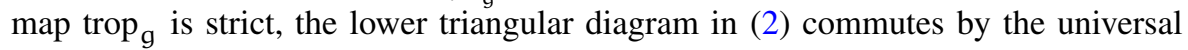
property from Proposition 3.13.

In the second step we notice that by construction of $\mathcal{T}_{\mathrm{g}}^{\mathrm{log}}$ as a fiber product $\mathcal{M}_{\mathrm{g}}^{\text {log }} \times{ }_{\mathcal{M}_{\mathrm{g}}^{\text {trop }}} \mathcal{T}_{\mathrm{g}}^{\text {trop }}$, we also have the following facts:

- The logarithmic strata of $\mathcal{T}_{\mathrm{g}}^{\mathrm{log}}$ are in a natural one-to-one correspondence with pairs consisting of a stable weighted graph of genus $g$ and a topological equivalence class of Teichmüller markings;

- An étale specialization $\eta_{\mathrm{G},[\phi]} \rightsquigarrow \eta_{\mathrm{G}^{\prime},\left[\phi^{\prime}\right]}$ of generic points of boundary strata that is not an isomorphism corresponds to a weighted edge contraction $G \rightarrow G^{\prime}$ that makes the resulting diagram of Teichmüller markings commute up to inner automorphisms.

- Both the monodromy group and the automorphism group of $(\mathrm{G},[\phi])$ are trivial.

Thus there is a natural equivalence between $\mathcal{T}_{\mathrm{g}}^{\text {trop }}$ and the $\mathcal{C}_{\mathcal{T}_{\mathrm{g}}^{\text {log }}}$. Since the tropicalization map trop $\mathrm{g}_{\mathrm{g}}$ is strict, as a base change of a strict tropicalization map, the upper triangular diagram is commutative by the universal property in Proposition 3.13. 
Finally, we observe that the two back squares in (2) commute by construction and thus the front square commutes, since trop $\mathrm{g}_{\mathrm{g}}$ is surjective.

\section{Skeletons and tropicalization}

Throughout this section let $\mathrm{K}$ be an algebraically closed non-Archimedean field with valuation ring $R$. In this section we construct $\overline{\mathcal{S}}_{\mathrm{g}}$ by applying Raynaud's generic fiber functor to $\mathcal{S}_{\mathrm{g}}^{\log }$ and identify its non-Archimedean tropicalization map with a strong deformation retraction onto the skeleton.

\subsection{Extended (generalized) cone complexes}

Given a rational polyhedral cone $\sigma$, its canonical extension is defined to be

$$
\bar{\sigma}=\operatorname{Hom}\left(S_{\sigma}, \overline{\mathbb{R}}_{\geqslant 0}\right)
$$

Here we write $\overline{\mathbb{R}}_{\geqslant 0}$ for the additive monoid $\overline{\mathbb{R}}_{\geqslant 0}=\mathbb{R}_{\geqslant 0} \sqcup\{\infty\}$. One may think of $\bar{\sigma}$ as a compactification of $\sigma$ given by adding further faces at infinity (see e.g. [64, Section 5] for details). As in $[7,75]$, we define the canonical extension $\bar{\Sigma}$ of a (generalized) cone complex $\Sigma$ as the colimit of the diagram that arises when we replace all cones in the defining diagram by their canonical extensions.

In the following we write $M_{\mathrm{g}}^{\text {trop }}$ and $\mathrm{T}_{\mathrm{g}}^{\text {trop }}$ for the coarse moduli spaces of $\mathcal{M}_{\mathrm{g}}^{\text {trop }}$ and $\mathcal{T}_{\mathrm{g}}^{\text {trop }}$ in the sense of Sect. 1.3 respectively. Both $M_{\mathrm{g}}^{\text {trop }}$ and $T_{\mathrm{g}}^{\text {trop }}$ are objects in the 2-category of cone stacks with faithful monodromy and in the category of generalized cone complexes. Denote by $\bar{M}_{\mathrm{g}}^{\text {trop }}$ and $\overline{\mathrm{T}}_{\mathrm{g}}^{\text {trop }}$ their canonical extensions. The points of $M_{\mathrm{g}}^{\text {trop }}$ are in natural one-to-one correspondence with stable tropical curves of genus $\mathrm{g}$ with real edge lengths and the points of $\mathrm{T}_{\mathrm{g}}^{\text {trop }}$ are pairs consisting of a stable tropical curve of genus $g$ and a topological equivalence class of Teichmüller markings. Their canonical extensions parametrize the same data, only we allow the edge lengths of the tropical curves to take non-zero values in the additive monoid $\overline{\mathbb{R}}_{\geqslant 0}=\mathbb{R}_{\geqslant 0} \sqcup\{\infty\}$ (see [7, Section 4] for details). Outer space $\mathrm{CV}_{\mathrm{g}}^{\text {trop }}$ in the sense of Culler-Vogtmann [28] is the locus of metric graphs in $T_{\mathrm{g}}^{\text {trop }}$, i.e. as the locus of tropical curves with all vertex weights equal to zero.

\subsection{Non-Archimedean tropicalization of $\overline{\mathcal{M}}_{\mathrm{g}}$}

Denote by $\overline{\mathcal{M}}_{\mathrm{g}}^{\text {an }}$ the non-Archimedean analytic stack associated to $\overline{\mathcal{M}}_{\mathrm{g}, \mathrm{K}}$. We refer the reader to [76,80] for the basic definitions of non-Archimedean analytic stacks and implicitly identify $\overline{\mathcal{M}}_{\mathrm{g}}^{\text {an }}$ with its underlying topological space, as introduced in [76, Section 3].

There is a natural non-Archimedean tropicalization map

$$
\operatorname{trop}_{\mathrm{g}}: \overline{\mathcal{M}}_{\mathrm{g}}^{\mathrm{an}} \longrightarrow \overline{\mathrm{M}}_{\mathrm{g}}^{\mathrm{trop}}
$$


that associates to a point in $\overline{\mathcal{M}}_{\mathrm{g}}^{\mathrm{an}}$, corresponding to a stable curve $\mathrm{X}$ over a nonArchimedean extension L of $\mathrm{K}$, its dual tropical curve $\Gamma_{\mathrm{X}}$. Let us explain this:

The valuative criterion for properness, applied to $\overline{\mathcal{M}}_{\mathrm{g}}$ tells us that there is a finite extension $\mathrm{L}^{\prime}$ of $\mathrm{L}$ such that the base change $\mathrm{X}_{\mathrm{L}^{\prime}}$ admits a stable model $\mathcal{X}$ over the valuation ring $\mathrm{R}^{\prime}$ of $\mathrm{L}^{\prime}$. In other words, there is a proper and flat scheme $\mathcal{X}$ over $\mathrm{R}^{\prime}$ with reduced fibers of dimension one such that the generic fiber is isomorphic to $X_{L^{\prime}}$ and the special fiber $\mathcal{X}_{0}$ is a stable nodal curve over the residue field of $\mathrm{R}^{\prime}$. The dual graph $\mathrm{G}_{\mathcal{X}_{0}}$ of $\mathcal{X}_{0}$ is the graph with vertices are the irreducible components of $\mathcal{X}_{0}$ and an edge between two vertices for every node connecting the corresponding components. A vertex weight $h: V\left(G_{\mathcal{X}_{0}}\right) \rightarrow \mathbb{Z}_{\geqslant 0}$ on $G_{\mathcal{X}_{0}}$ associates to a vertex the genus of the normalization of the corresponding component. Étale locally around every node, the scheme $\mathcal{X}$ is given by $x y=r_{e}$ for two coordinates $x$ and $y$ and an element $r \in R^{\prime}$. The edge length on $G_{\mathcal{X}_{0}}$ is given by $|e|=\operatorname{val}\left(r_{e}\right)$. Notice hereby that for the edges $e$ corresponding to nodes that were already present in the generic fiber, we always have $|e|=0$. The dual tropical curve $\Gamma_{X}$ is the tropical curve given by the tuple $\left(\mathrm{G}_{\mathcal{X}_{0}}, \mathrm{~h},||.\right)$ (with edge lengths in $\overline{\mathbb{R}}_{\geqslant 0}$ ).

It follows a posteriori from the identification of this map with the strong deformation

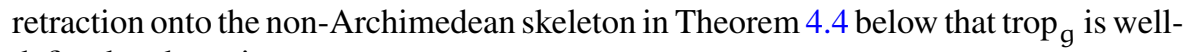
defined and continuous.

\subsection{Raynaud's generic fiber functor}

Berkovich analytification defines a functor from the category of schemes locally of finite type over $K$ to the category of non-Archimedean analytic spaces. By [15] a scheme $X$ locally of finite type over $K$ is separated if and only $X^{a n}$ is a Hausdorff space. Since we are considering the non-separated stack $\mathcal{S}_{\mathrm{g}}^{\mathrm{log}}$, we therefore want to work with a different analytification functor, known as Raynaud's generic fiber functor (as introduced in [16]).

It associates to a flat scheme $\mathscr{X}$ locally of finite type over $\mathrm{R}$ a Berkovich analytic space $\mathscr{X}_{\eta}$ that functions as an analytic generic fiber of the formal completion of $\mathscr{X}$ along the maximal ideal of R. Suppose that $\mathscr{X}=$ Spec $\mathscr{A}$ is affine and write $X=\operatorname{Spec} A$ for its generic fiber, where $A=\mathscr{A} \otimes_{R} K$. In this case, the Raynaud generic fiber is the affinoid domain in $X^{\text {an }}$ whose points are those seminorms $|\cdot|_{x}$ on A for which $|a|_{x} \leqslant 1$ for all $a \in \mathscr{A}$, i.e. those seminorms that extend to a bounded seminorm on $\mathscr{A}$.

In general, when $\mathscr{X}$ is not affine, the Raynaud generic fiber $\mathscr{X}_{\eta}$ is given by glueing the affine patches of $\mathscr{X}$. A point in $\mathscr{X}_{\eta}$ may be represented by a morphism Spec $\mathrm{R}^{\prime} \rightarrow$ $\mathscr{X}$ from a valuation ring $\mathrm{R}^{\prime}$ that extends $\mathrm{R}$. Two morphisms $\operatorname{Spec} \mathrm{R}^{\prime} \rightarrow \mathscr{X}$ and Spec $\mathrm{R}^{\prime \prime} \rightarrow \mathscr{X}$ represent the same point if and only if there is a valuation ring $\Omega$ that extends both $R^{\prime}$ and $R^{\prime \prime}$ such that the induced diagram

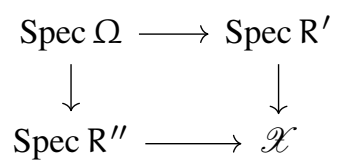


commutes. By the valuative criteria, the Raynaud generic fiber $\mathscr{X}_{\eta}$ is an analytic domain in the Berkovich analytic space $\mathscr{X}_{\mathrm{K}}^{\mathrm{an}}$ associated to the algebraic geometric generic fiber $\mathscr{X}_{\mathrm{K}}$ of $\mathscr{X}$ if and only if $\mathscr{X}$ is separated over $\mathrm{R}$, and $\mathscr{X}_{\eta}$ is isomorphic to $\mathscr{X}_{\mathrm{K}}^{\mathrm{an}}$ if and only if $\mathscr{X}$ is proper over $\mathrm{R}$.

In [80], Yu has extended the generic fiber functor $(.)_{\eta}$ to a functor

$$
(.)_{\eta}: \text { Alg.Stacks }_{\text {flat, loc.f.t. } / k} \longrightarrow \text { An.Stacks }_{k}
$$

such that, whenever $\mathscr{X}=[\mathscr{U} / \mathscr{R}]$ is a groupoid presentation of an algebraic stack $\mathscr{X}$ that is flat and locally of finite type over $\mathrm{R}$, we have

$$
\mathscr{X}_{\eta} \simeq\left[\mathscr{U}_{\eta} / \mathscr{R}_{\eta}\right]
$$

Again a point in $\mathscr{X}_{\eta}$ may be represented by a morphism Spec $\mathrm{R}^{\prime} \rightarrow \mathscr{X}$ from a valuation ring $\mathrm{R}^{\prime}$ that extends $\mathrm{R}$. Two morphisms $\operatorname{Spec} \mathrm{R}^{\prime} \rightarrow \mathscr{X}$ and $\operatorname{Spec} \mathrm{R}^{\prime \prime} \rightarrow \mathscr{X}$ then represent the same point if and only if there is a valuation ring $\Omega$ that extends both $R^{\prime}$ and $R^{\prime \prime}$ such that the induced diagram

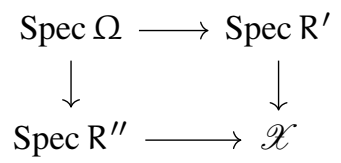

is 2-commutative. Yet again, by the valuative criteria, the Raynaud generic fiber $\mathscr{X}_{\eta}$ is an analytic domain in the Berkovich analytic stack $\mathscr{X}_{\mathrm{K}}^{\mathrm{an}}$ if and only if $\mathscr{X}$ is separated over $\mathrm{R}$, and $\mathscr{X}_{\eta}$ is equivalent to $\mathscr{X}_{\mathrm{K}}^{\mathrm{an}}$ if and only if $\mathscr{X}$ is proper over $\mathrm{R}$.

\subsection{Non-Archimedean Teichmüller space and its tropicalization}

Recall from Sect. 3.4 that $\underline{\mathcal{T}}_{\mathrm{g}}^{\text {log }}$ is the underlying algebraic stack of $\mathcal{T}_{\mathrm{g}}^{\text {log }}=$ $\mathcal{M}_{\mathrm{g}}^{\text {log }} \times_{\mathcal{M}_{\mathrm{g}}^{\text {trop }}} \mathcal{T}_{\mathrm{g}}^{\text {trop }}$. It is a smooth and universally closed, but not separated DeligneMumford stack over Spec $\mathbb{Z}$. We write $\underline{\mathcal{T}}_{\mathrm{g}, \mathrm{R}}^{\mathrm{log}}$ for the base change of $\underline{\mathcal{T}}_{\mathrm{g}}^{\text {log }}$ to the valuation ring $R$.

Definition 4.1 The extended non-Archimedean Teichmüller space $\overline{\mathcal{T}}_{\mathrm{g}}$ over $\mathrm{K}$ is defined to be

$$
\overline{\mathcal{T}}_{\mathrm{g}}=\underline{\mathcal{T}}_{\mathrm{g}, \mathrm{R}, \eta}^{\mathrm{log}} .
$$

A point in $\overline{\mathcal{T}}_{\mathrm{g}}$ is represented by a tuple $(X, \phi)$ where $\mathrm{X}$ is a stable curve of genus $g$ over a non-Archimedean extension $L$ of $K$ together with a Teichmüller marking of the dual tropical curve $\Gamma_{X}$ of its stable reduction. It is well-known that the geometric realization $\left|\Gamma_{X}\right|$ of $\Gamma_{X}$ as a metric space arises as the minimal skeleton of the nonArchimedean analytic space $X_{L}^{a n}$ (see $[15,18]$ for details). A Teichmüller marking on 
$\Gamma_{X}$ amounts to the choice of an equivalence $\pi_{1}\left(X^{a n}\right)=\pi_{1}\left(\left|\Gamma_{X}\right|\right) \stackrel{\sim}{\rightarrow} F_{b_{1}}$ where $b_{1}$ is the Betti number of both $X^{a n}$ and its minimal skeleton $\left|\Gamma_{X}\right|$. So, in particular, we have a natural non-Archimedean tropicalization map

$$
\begin{gathered}
\operatorname{trop}_{\mathrm{g}}: \overline{\mathcal{T}}_{\mathrm{g}} \longrightarrow \overline{\mathrm{T}}_{\mathrm{g}}^{\text {trop }} \\
\left(\mathrm{X}, \phi: \pi_{1}\left(\mathrm{X}^{\mathrm{an}}\right) \stackrel{\sim}{\longrightarrow} \mathrm{F}_{\mathrm{b}_{1}}\right) \longmapsto\left(\Gamma_{\mathrm{X}}, \phi: \pi_{1}\left(\left|\Gamma_{\mathrm{X}}\right|\right) \stackrel{\sim}{\longrightarrow} \mathrm{F}_{\mathrm{b}_{1}}\right) .
\end{gathered}
$$

We define $\mathcal{T}_{\mathrm{g}}$ as the locus of pairs $(\mathrm{X}, \phi)$ where $\mathrm{X}$ is a smooth curve and refer to it as the non-Archimedean Teichmüller space over K. It also arises as the preimage $\operatorname{trop}_{\mathrm{g}}^{-1}\left(\mathrm{~T}_{\mathrm{g}}^{\mathrm{trop}}\right)$ of the locus of non-extended tropical curves (with edge lengths only in $\mathbb{R}_{\geqslant 0}$ instead of $\left.\overline{\mathbb{R}}_{\geqslant 0}=\mathbb{R}_{\geqslant 0} \sqcup\{\infty\}\right)$ and as the preimage of the analytification $\mathcal{M}_{\mathrm{g}}^{\mathrm{an}}$ of $\mathcal{M}_{\mathrm{g}}$ under the natural map $\overline{\mathcal{T}}_{\mathrm{g}} \rightarrow \overline{\mathcal{M}}_{\mathrm{g}}^{\mathrm{an}}$ that forgets the marking.

Proof of Theorem A Since $\underline{\mathcal{T}}_{\mathrm{g}}^{\mathrm{log}}$ is smooth over $\mathbb{Z}$, its base change to $\mathrm{R}$ is smooth over $\mathrm{R}$ and therefore the Raynaud generic fiber is $\mathrm{G}$-smooth over $\mathrm{K}$. Let $\mathrm{G}$ be stable weighted graph of genus $g$ and consider the rational polyhedral cone $\sigma_{G}=\mathbb{R}_{\geqslant 0}^{E(G)}$, which parametrizes all tropical curves whose underlying graph is a weighted edge contraction of $G$. Write $U_{\sigma_{G}}$ for the affine toric variety Spec $\mathbb{Z}\left[S_{\sigma_{G}}\right]$ associated to $\sigma_{G}$. There is a natural morphism $\sigma_{\mathrm{G}} \rightarrow \mathcal{M}_{\mathrm{g}}^{\text {trop }}$ that induces a morphism $\mathrm{U}_{\sigma} \rightarrow \mathcal{M}_{\mathrm{g}}^{\text {trop }}$ (here the right hand side is the really the Artin fan $\mathcal{A}_{\mathcal{M}_{\mathrm{g}}^{\text {trop }}}$ ). The base change

$$
\widetilde{\mathrm{U}}_{\sigma_{\mathrm{G}}}=\mathrm{U}_{\sigma_{\mathrm{G}}} \times_{\mathcal{M}_{\mathrm{g}}^{\text {trop }}} \mathcal{T}_{\mathrm{g}}^{\text {trop }}
$$

is a non-separated toric variety. Each of its maximal torus-invariant open affine subsets is isomorphic to $\mathrm{U}_{\sigma_{\mathrm{G}}}$, since $\mathcal{T}_{\mathrm{g}}^{\text {trop }} \rightarrow \mathcal{M}_{\mathrm{g}}^{\text {trop }}$ is a strict cover. Thus, applying the Raynaud generic fiber functor, we obtain a morphism $\widetilde{U}_{\sigma_{G}, R, \eta} \rightarrow U_{\sigma_{G}, R, \eta}$ without boundary. Since $\overline{\mathcal{T}}_{\mathrm{g}} \rightarrow \overline{\mathcal{M}}_{\mathrm{g}}^{\mathrm{an}}$ arises étale locally as a base change of such morphisms, it also without boundary. This proves that $\overline{\mathcal{T}}_{\mathrm{g}}$ is without boundary over $\mathrm{K}$, since $\overline{\mathcal{M}}_{\mathrm{g}}^{\mathrm{an}}$ is. Since $\overline{\mathcal{T}}_{\mathrm{g}}$ is G-smooth and without boundary, it is also smooth.

In order to show that $\overline{\mathcal{T}}_{\mathrm{g}}$ is separated we show that the diagonal morphism $\Delta: \overline{\mathcal{T}}_{\mathrm{g}} \rightarrow$ $\overline{\mathcal{T}}_{\mathrm{g}} \times \overline{\mathcal{T}}_{\mathrm{g}}$ is proper. Let $\mathrm{U} \rightarrow \overline{\mathcal{T}}_{\mathrm{g}} \times \overline{\mathcal{T}}_{\mathrm{g}}$ be a morphism from a strict analytic space $\mathrm{U}$. This corresponds to a flat and proper analytic family $X \rightarrow U$ of stable curves together with a family of Teichmüller markings of the dual tropical curves, compatible with étale specialization. The fiber product $\overline{\mathcal{T}}_{\mathrm{g}} \times \overline{\mathcal{T}}_{\mathrm{g}} \times \overline{\mathcal{T}}_{\mathrm{g}} \mathrm{U}$ is representable by an analytic space $\mathrm{V}$ whose points are exactly the triples $(x, y, \phi)$ consisting of two points $x, y \in U$ and an isomorphism $\phi: X_{x} \stackrel{\sim}{\rightarrow} X_{y}$ that is compatible with the Teichmüller markings on the dual tropical curves. Since the diagonal morphism $\overline{\mathcal{M}}_{\mathrm{g}}^{\mathrm{an}} \rightarrow \overline{\mathcal{M}}_{\mathrm{g}}^{\mathrm{an}} \times \overline{\mathcal{M}}_{\mathrm{g}}^{\mathrm{an}}$ is proper, the base change of $\mathrm{V} \rightarrow \mathrm{U}$ to any affinoid domain in $\mathrm{U}$ is finite and this implies that $\mathrm{V} \rightarrow \mathrm{U}$ is without boundary. Any compact subset $A$ in $\mathrm{U}$ will be a subset of a finite union of affinoid domains and thus this also implies that the preimage of $A$ in $\mathrm{V}$ is compact. Therefore $\mathrm{V} \rightarrow \mathrm{U}$ is a proper morphism and, since $\mathrm{U}$ was chosen arbitrarily, this implies that the diagonal morphism $\Delta: \overline{\mathcal{T}}_{\mathrm{g}} \rightarrow \overline{\mathcal{T}}_{\mathrm{g}} \times \overline{\mathcal{T}}_{\mathrm{g}}$ is proper. 
Proof of Theorem $D$ This immediately follows from Theorem 3.10 and the fact that both the functors (.) $\otimes$ Spec $R$ and (.) $\eta$ preserve (2-)colimits.

\subsection{Non-Archimedean skeletons of stacky normal crossing pairs with good reduction}

Let $(\mathscr{X}, \mathscr{D})$ be a strictly semistable pair over $\mathrm{R}$, consisting of a flat scheme $\mathscr{X}$ locally of finite type over Spec R whose generic fiber is smooth and whose special fiber has strict normal crossings and an effective strict normal crossing divisor $\mathscr{D}=$ $\mathcal{D}_{1}+\cdots+\mathcal{D}_{s}$ on $\mathscr{X}$ which includes the special fiber. In [34, Section 4] the authors expand on a construction of Berkovich in [17] and show that the generic fiber $\mathscr{X}_{\eta}$ admits a strong deformation retraction $\rho_{(\mathscr{X}, \mathscr{D})}: \mathscr{X}_{\eta} \rightarrow \mathscr{X}_{\eta}$ onto a closed subset $\bar{\Sigma}(\mathscr{X}, \mathscr{D})$ of $\mathscr{X}_{\eta}$, the skeleton of the strictly semistable pair $(\mathscr{X}, \mathscr{D})$.

Remark 4.2 In [34] the authors always require that $\mathscr{X}$ be proper over $\mathrm{R}$. This assumption is not necessary, if we work with the Raynaud generic fiber $\mathscr{X}_{\eta}$ instead of the Berkovich analytic space of the algebraic generic fiber of $\mathscr{X}$.

For the purpose of this article we assume that $\mathscr{X}$ is smooth over R, i.e. that $\mathscr{X}$ has good reduction. In this case, the skeleton $\bar{\Sigma}(\mathscr{X}, \mathscr{D})$ canonically has the structure of an extended cone complex: The space $\mathcal{X}$ is naturally stratified by locally closed subschemes; the strata are the connected components of the smooth locus of $\mathcal{D}_{i_{1}} \cap \cdots \cap$ $\mathcal{D}_{i_{k}}$. If $\mathscr{U} \subseteq \mathscr{X}$ is small open subset of $\mathscr{X}$ (or a building block in the terminology of [34, Section 4.3]), i.e. if it contains a unique closed stratum, the skeleton is naturally homeomorphic to the extended cone $\bar{\sigma}_{\mathscr{X}}=\operatorname{Hom}\left(\bar{M}_{\mathscr{D}}, \overline{\mathbb{R}}_{\geqslant 0}\right)$ and the retraction $\rho_{(\mathscr{X}, \mathscr{D})}$ is given by

$$
x \longmapsto\left(\bar{M}_{\mathscr{D}} \rightarrow \mathcal{O}_{\mathscr{U}} / \mathcal{O}_{\mathscr{U}}^{*} \stackrel{-\log |\cdot|_{x}}{\longrightarrow} \overline{\mathbb{R}}_{\geqslant 0}\right)
$$

Here $M_{\mathscr{D}}$ denotes the natural divisorial logarithmic structure on $\mathscr{X}$ associated to the divisor $\mathscr{D}$, i.e. the sheaf of sections of $s \in \mathcal{O}_{X}$ such that $\left.s\right|_{\mathscr{U}-\mathscr{D}} \in \mathcal{O}_{\mathcal{X}}^{*}$; this way $\bar{M}_{\mathscr{D}}=M_{\mathscr{D}} / \mathcal{O}_{\mathscr{X}}^{*}$ is identified with the sheaf of effective Cartier divisors with support in $\mathscr{D}$. In general, for every strictly semistable pair $(\mathscr{X}, \mathscr{D})$ there is a cover by small open subsets $\mathscr{U}_{i}$ as well as a cover $\mathscr{V}_{k}^{i j}$ of $\mathscr{U}_{i} \cap \mathscr{U}_{j}$ by small open subsets $\mathscr{V}_{k}^{i j}$. Then the extended cone complex $\bar{\Sigma}(\mathscr{X}, \mathscr{D})$ is the colimit of the induced diagram of proper face morphisms $\bar{\sigma}_{V_{k}^{i j} \hookrightarrow} \bar{\sigma}_{U_{i}}$ and the retraction map is defined by (3) on every small open subset $\mathscr{U} \subseteq \mathscr{X}$.

In the following Lemma 4.3, we generalize this construction to the case of a stacky normal crossing pair $(\mathscr{X}, \mathscr{D})$ with good reduction, i.e. to the case of a DeligneMumford stack $\mathscr{X}$ that is smooth over Spec R together with an effective Cartier divisor $\mathscr{D}$ on $\mathscr{X}$ that has (stack-theoertically) normal crossings. We say that an étale morphism $\mathrm{f}: \mathscr{U} \rightarrow \mathscr{X}$ is small, if $\left(\mathscr{U}, f^{*} \mathscr{D}\right)$ is a strictly semistable pair with good reduction that is small.

Lemma 4.3 Let $\mathscr{X}$ be a Deligne-Mumford stack that is smooth over $\mathrm{R}$ and let $\mathscr{D}$ be an effective Cartier divisor on $\mathscr{X}$ with (stack-theoretically) normal crossings. Then 
there is a strong deformation retraction $\rho_{(\mathscr{X}, \mathscr{D})}: \mathscr{X}_{\eta} \rightarrow \mathscr{X}_{\eta}$ onto a closed subset $\bar{\Sigma}(\mathscr{X}, \mathscr{D})$, the non-Archimedean skeleton associated to $(\mathscr{X}, \mathscr{D})$. The skeleton $\bar{\Sigma}(\mathscr{X}, \mathscr{D})$ is naturally homeomorphic to the extended generalized cone complex associated to $\mathcal{C}(\mathscr{X}, \mathscr{D})$ and, on a small étale neighborhood $\mathscr{U} \rightarrow \mathscr{X}$, the retraction map $\rho_{(\mathscr{X}, \mathscr{D})}$ is given by the non-Archimedean tropicalization map

$$
\begin{gathered}
\mathscr{U}_{\eta} \longrightarrow \bar{\sigma}_{\mathscr{U}}=\operatorname{Hom}\left(\bar{M}_{\mathscr{U}}, \overline{\mathbb{R}}_{\geqslant 0}\right) \\
x \longmapsto\left(\bar{M}_{\mathscr{U}} \rightarrow \mathcal{O}_{\mathscr{U}} / \mathcal{O}_{\mathscr{U}}^{*} \stackrel{-\log |\cdot|_{x}}{\longrightarrow} \overline{\mathbb{R}}_{\geqslant 0}\right) .
\end{gathered}
$$

Proof Consider the category $\mathrm{Q}(\mathscr{X}, \mathscr{D})$ whose objects are small étale morphisms f: $\mathscr{U} \rightarrow \mathscr{X}$ and whose morphisms are commutative diagrams

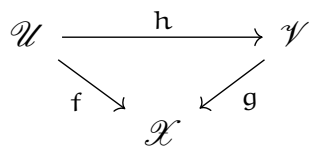

such that $\mathrm{f}^{*} \mathscr{D}=(\mathrm{g} \circ \mathrm{h})^{*} \mathscr{D}$. There is a natural functor $\mathrm{Q}(\mathscr{X}, \mathscr{D}) \rightarrow \mathbf{R P C}$ that is given by $\mathscr{U} \mapsto \sigma_{\mathrm{U}}$. Denote by $\mathrm{M}_{\mathscr{D}}$ the divisorial logarithmic structure on $\mathscr{X}$ associated to $\mathscr{D}$ and note that $\left(\mathscr{X}, M_{\mathscr{D}}\right)$ is logarithmically smooth over Spec $\mathrm{R}$ (with the trivial logarithmic structure). The generalized cone complex associated to $\mathcal{C}\left(\mathscr{X}, M_{\mathscr{D}}\right)$ is precisely the colimit over the diagram of all $\sigma_{\mathscr{U}}$ in $\mathrm{Q}(\mathscr{X}, \mathscr{D})$, since every geometric point has of $\mathscr{X}$ has a small open étale neighborhood, in which it is in the deepest stratum.

The underlying topological space of $\mathscr{X}_{\eta}$ is the colimit of all $\mathrm{f}_{\eta}: \mathscr{U}_{\eta} \rightarrow \mathscr{X}_{\eta}$ for $\mathrm{f}: \mathscr{U} \rightarrow \mathscr{X}$ in $\mathrm{Q}(\mathscr{X}, \mathscr{D})$. Moverover, for every diagram (4) the induced diagram of retraction maps

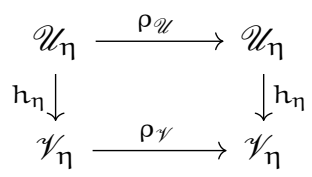

is commutative. So the $\rho_{\mathscr{U}}$ descend to a retraction map $\mathscr{X}_{\eta} \rightarrow \mathscr{X}_{\eta}$, whose image is defined to be the skeleton $\bar{\Sigma}(\mathscr{X}, \mathscr{D})$ of $\mathscr{X}$. It is precisely the colimit of all $\bar{\sigma}_{\mathscr{U}}$, taken over the small étale neighborhoods $\mathrm{f}: \mathscr{U} \rightarrow \mathscr{X}$ in $\mathrm{Q}(\mathscr{X}, \mathscr{D})$ and therefore isomorphic to the canonical extension of the generalzed cone complex associated to $\mathcal{C}\left(\mathscr{X}, M_{\mathscr{D}}\right)$.

In order to show that there is a strong homotopy equivalence between $\rho_{\mathscr{X}}$ and the identity on $\mathscr{X}_{\eta}$, we need to observe that the induced diagram of homotopies on (4) is commutative. This argument has already been carried out in [73, Section 3.3] and [7, Proposition 6.1.4] over base fields with trivial absolute; it carries over to our situation without any changes. 


\subsection{Skeletons of $\overline{\mathcal{T}}_{\mathrm{g}}$ and $\overline{\mathcal{M}}_{\mathrm{g}}^{\mathrm{an}}$}

The following Theorem 4.4 expands on Theorem B from the introduction and generalizes the main result of [7] to the case of a non-trivially valued base field.

Theorem 4.4 The skeleton of $\overline{\mathcal{M}}_{\mathrm{g}}^{\mathrm{an}}$ is isomorphic to $\overline{\mathrm{M}}_{\mathrm{g}}^{\text {trop }}$ and the skeleton of $\overline{\mathcal{T}}_{\mathrm{g}}$ is isomorphic to $\overline{\mathcal{T}}_{\mathrm{g}}^{\text {trop }}$ so that the natural diagram

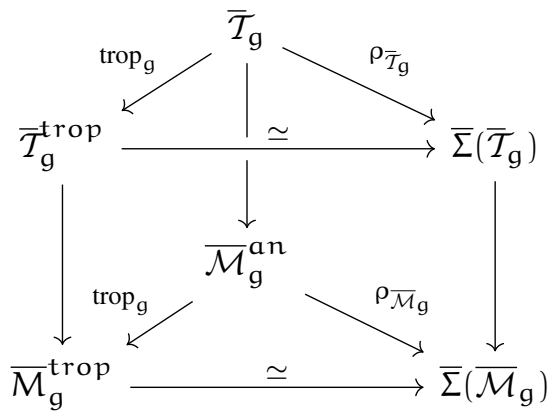

\section{commutes.}

Proof of Theorem 4.4 Use Theorem 3.14 together with Lemma 4.3 applied to $\overline{\mathcal{M}}_{\mathrm{g}}^{\mathrm{an}}$ and $\overline{\mathcal{T}}_{\mathrm{g}}$. The commutativity of diagram (5) follows form the commutativity of diagram (2) in Theorem 3.14.

Proof of Corollary $C$ For a stacky normal crossing pair $(\mathcal{X}, \mathcal{D})$, the homotopy between $\rho_{(\mathcal{X}, \mathcal{D})}$ and identity preserves the fibers of $\rho_{(\mathcal{X}, \mathcal{D})}$; this may be checked étale locally only toric varieties, on which the homotopy is defined via the torus operation. Using Theorem 4.4, we may therefore restrict the homotopy to the locus of smooth Mumford curves in $\overline{\mathcal{T}}_{\mathrm{g}}$ and find that the tropicalization defines a strong deformation retraction onto Culler-Vogtmann Outer space $\mathrm{CV}_{\mathrm{g}}$.

Remark 4.5 Denote by $\overline{\mathrm{CV}}_{\mathrm{g}}$ the canonical extension of Culler-Vogtmann Outer space, where we allow the edge lengths of graphs to attain value $\infty$ (i.e. the closure of $\mathrm{CV}_{g}$ in $\overline{\mathrm{T}}_{\mathrm{g}}^{\text {trop }}$ ). The same argument as in the proof of Corollary $\mathrm{C}$ shows that $\overline{\mathrm{CV}}_{\mathrm{g}}$ is a strong deformation retract of the locus $\overline{\mathcal{T}}_{\mathrm{g}}^{\text {Mum }}$ of stable Mumford curves in $\overline{\mathcal{T}}_{\mathrm{g}}$.

Acknowledgements The idea for this project was born during the Summer School "Around Moduli Spaces" that took place at Saarland University in September 2019, where Frank Herrlich gave a minicourse on "Schottky groups and moduli spaces". We thank him for his crystal clear lectures and answering several of our questions; we also thank the organizers Gabriele Weitze-Schmidthüsen, Christian Steinhart, and Andrea Thevis for creating this opportunity. Thanks are also due to Matt Baker, Charles Frohman, Martin Möller, Felix Röhrle, Farbod Shokrieh, and Filippo Viviani for useful discussions and remarks related to this manuscript. The author would, in particular, like to thank Annette Werner; she was part of this project in the beginning, but decided to not be a coauthor. We thank Daniele Turchetti and Jérôme Poineau for our communication concerning their project [63] and for pointing out a crucial difference between GerritzenHerrlich's Schottky space and $\overline{\mathcal{T}}_{\mathrm{g}}$, as well as Hannah Markwig and Christian Steinhart for keeping him in the 
loop on an ongoing project on how to tropicalize Archimedean Teichmüller space. Finally, we acknowledge support from the LOEWE-Schwerpunkt "Uniformisierte Strukturen in Arithmetik und Geometrie".

Funding Open Access funding enabled and organized by Projekt DEAL.

Open Access This article is licensed under a Creative Commons Attribution 4.0 International License, which permits use, sharing, adaptation, distribution and reproduction in any medium or format, as long as you give appropriate credit to the original author(s) and the source, provide a link to the Creative Commons licence, and indicate if changes were made. The images or other third party material in this article are included in the article's Creative Commons licence, unless indicated otherwise in a credit line to the material. If material is not included in the article's Creative Commons licence and your intended use is not permitted by statutory regulation or exceeds the permitted use, you will need to obtain permission directly from the copyright holder. To view a copy of this licence, visit http://creativecommons.org/licenses/by/4.0/.

\section{References}

1. Arbarello, E., Cornalba, M., Griffiths, P.A.: Geometry of algebraic curves. Volume II, Grundlehren der Mathematischen Wissenschaften [Fundamental Principles of Mathematical Sciences], vol. 268, Springer, Heidelberg, 2011, With a contribution by Joseph Daniel Harris

2. Abramovich, D., Chen, Q., Gillam, D., Huang, Y., Olsson, M., Satriano, M., Sun, S.: Logarithmic geometry and moduli, Handbook of moduli. Vol. I, Adv. Lect. Math. (ALM), vol. 24, Int. Press, Somerville, MA, 2013, pp. 1-61

3. Abikoff W.: Degenerating families of Riemann surfaces. Ann. Math. (2) 105 (1), 29-44 (1977). https:// doi.org/10.2307/1971024

4. Abramovich, D., Chen, Q., Gross, M., Siebert, B.: Decomposition of degenerate Gromov-Witten invariants. Compos. Math. 156(10), 2020-2075 (2020)

5. Abramovich, D., Chen, Q., Marcus, S., Ulirsch, M., Wise, J.: Skeletons and fans of logarithmic structures. Nonarchimedean and tropical geometry, Simons Symp., Springer, [Cham], pp. 287-336 (2016)

6. Abramovich, D., Chen, Q., Marcus, S., Wise, J.: Boundedness of the space of stable logarithmic maps. J. Eur. Math. Soc. (JEMS) 19(9), 2783-2809 (2017)

7. Abramovich, D., Caporaso, L., Payne, S.: The tropicalization of the moduli space of curves. Ann. Sci. Éc. Norm. Supér (4) 48(4), 765-809 (2015)

8. André, Y.: Period mappings and differential equations. From $\mathbb{C}$ to $\mathbb{C}_{p}$, MSJ Memoirs, vol. 12, Mathematical Society of Japan, Tokyo: Tohoku-Hokkaido lectures in arithmetic geometry. With appendices by F. Kato and N, Tsuzuki (2003)

9. Abramovich, D., Olsson, M., Vistoli, A.: Twisted stable maps to tame Artin stacks. J. Algebraic Geom. 20(3), 399-477 (2011)

10. Abreu, A., Pacini, M.: The universal tropical Jacobian and the skeleton of the Esteves universal Jacobian. Proc. Lond. Math. Soc. 120(3), 328-369 (2020)

11. Abreu, A., Pacini, M.: The resolution of the universal Abel map via tropical geometry and applications. Adv. Math. 378, 107520 (2021)

12. Abramovich, D., Wise, J.: Birational invariance in logarithmic Gromov-Witten theory. Compos. Math. 154(3), 595-620 (2018)

13. Bass, H.: Covering theory for graphs of groups. J. Pure Appl. Algebra 89(1-2), 3-47 (1993)

14. Bainbridge, M., Chen, D., Gendron, Q., Grushevsky, S., Möller, M.: The moduli space of multi-scale differentials. arXiv:1910.13492 [math] (2019)

15. Berkovich, V.G.: Spectral theory and analytic geometry over non-Archimedean fields, Mathematical Surveys and Monographs, vol. 33. American Mathematical Society, Providence, RI (1990)

16. Berkovich, V.G.: Vanishing cycles for formal schemes. II. Invent. Math. 125(2), 367-390 (1996)

17. Berkovich, V.G.: Smooth p-adic analytic spaces are locally contractible. Invent. Math. 137(1), 1-84 (1999)

18. Baker, M., Payne, S., Rabinoff, J.: Nonarchimedean geometry, tropicalization, and metrics on curves. Algebraic Geom. 3(1), 63-105 (2016)

19. Baker, M., Rabinoff, J.: The skeleton of the Jacobian, the Jacobian of the skeleton, and lifting meromorphic functions from tropical to algebraic curves. Int. Math. Res. Not. IMRN (2015), no. 16, 7436-7472 
20. Brandt, M., Ulirsch, M.: Symmetric powers of algebraic and tropical curves: a non-archimedean perspective. arXiv:1812.08740 [math] (2018)

21. Caporaso, L.: Algebraic and tropical curves: comparing their moduli spaces. Handbook of moduli. Vol. I, Adv. Lect. Math. (ALM), vol. 24, Int. Press, Somerville, MA, pp. 119-160 (2013)

22. Caporaso, L., Christ, K.: Combinatorics of compactified universal Jacobians. Adv. Math. 346, 10911136 (2019)

23. Cavalieri, R., Chan, M., Ulirsch, M., Wise, J.: A moduli stack of tropical curves, Forum Math. Sigma 8 Paper No. e23, 93 (2020)

24. Caporaso, L., Melo, M., Pacini, M.: Tropicalizing the moduli space of spin curves. Selecta Math. (N.S.) 26 (2020), no. 1, Paper No. 16

25. Cavalieri, R., Markwig, H., Ranganathan, D.: Tropicalizing the space of admissible covers. Math. Ann. 364(3-4), 1275-1313 (2016)

26. Chan, M., Melo, M., Viviani, F.: Tropical Teichmüller and Siegel spaces. Algebraic and combinatorial aspects of tropical geometry. Contemp. Math., vol. 589, American Mathematical Society, Providence, RI, pp. 45-85 (2013)

27. Conrad, B., Temkin, M.: Non-Archimedean analytification of algebraic spaces. J. Algebraic Geom. 18(4), 731-788 (2009)

28. Culler, M., Vogtmann, K.: Moduli of graphs and automorphisms of free groups. Invent. Math. 84(1), 91-119 (1986)

29. Fresnel, J., van der Put, M.: Rigid Analytic Geometry and its Applications, Progress in Mathematics, vol. 218. Birkhäuser Boston Inc, Boston (2004)

30. Gerritzen, L.: Die Jacobi-Abbildung über dem Raum der Mumfordkurven. Math. Ann. 261(1), 81-100 (1982)

31. Gerritzen, L.: p-adic Siegel halfspace. Study group on ultrametric analysis, 9th year: 1981/82, No. 3 (Marseille, 1982), Inst. Henri Poincaré, Paris, 1983, pp. Exp. No. J9, 7

32. Gerritzen, L.: p-adic Teichmüller space and Siegel halfspace. Study group on ultrametric analysis, 9 th year: 1981/82, No. 2, Inst. Henri Poincaré, Paris, 1983, pp. Exp. No. 26, 15

33. Gerritzen, L., Herrlich, F.: The extended Schottky space. J. Reine Angew. Math. 389, 190-208 (1988)

34. Gubler, W., Rabinoff, J., Werner, A.: Skeletons and tropicalizations. Adv. Math. 294, 150-215 (2016)

35. Hejhal, D.A.: On Schottky and Teichmüller spaces. Adv. Math. 15, 133-156 (1975)

36. Herrlich, F., Teichmüllerräume, N.: Nederl. Akad. Wetensch. Indag. Math. 49(2), 145-169 (1987)

37. Herrlich, F.: The extended Teichmüller space. Math. Z. 203(2), 279-291 (1990)

38. Herrlich, F.: The non-Archimedean extended Teichmüller space, p-adic analysis (Trento, : Lecture Notes in Math., vol. 1454. Springer, Berlin 1990, 256-266 (1989)

39. Herr, L.: The Log Product Formula. arXiv:1908.04936 [math] (2019)

40. Herrlich, F.: Personal communication (2019)

41. Holmes, D., Kass, J.L., Pagani, N.: Extending the double ramification cycle using Jacobians. Eur. J. Math. 4(3), 1087-1099 (2018)

42. Holmes, D.: Extending the double ramification cycle by resolving the Abel-Jacobi map. J. Inst. Math. Jussieu 1-29 (2019)

43. Ichikawa, T.: Generalized Tate curve and integral Teichmüller modular forms. Am. J. Math. 122(6), 1139-1174 (2000)

44. Kato, K.: Logarithmic structures of Fontaine-Illusie, Algebraic analysis, geometry, and number theory (Baltimore, MD, : Johns Hopkins University Press, Baltimore, MD 1989, 191-224 (1988)

45. Kato, K.: Toric singularities. Am. J. Math. 116(5), 1073-1099 (1994)

46. Kato, F.: Log smooth deformation and moduli of log smooth curves. Int. J. Math. 11(2), 215-232 (2000)

47. Kajiwara, T., Kato, K., Nakayama, C.: Logarithmic abelian varieties. I. Complex analytic theory. J. Math. Sci. Univ. Tokyo 15(1), 69-193 (2008)

48. Knudsen, F.F.: The projectivity of the moduli space of stable curves. II. The stacks $M_{g, n}$. Math. Scand. 52(2), 161-199 (1983)

49. Koebe, P.: Über die Uniformisierung der algebraischen Kurven. IV. Math. Ann. 75(1), 42-129 (1914)

50. Kass, J.L., Pagani, N.: The stability space of compactified universal Jacobians. Trans. Am. Math. Soc. 372(7), 4851-4887 (2019)

51. Len, Y., Ulirsch, M.: Skeletons of Prym varieties and Brill-Noether theory. arXiv:1902.09410 [math] (2019) 
52. Lütkebohmert, W.: Rigid geometry of curves and their Jacobians. Ergebnisse der Mathematik und ihrer Grenzgebiete. 3. Folge. A Series of Modern Surveys in Mathematics [Results in Mathematics and Related Areas. 3rd Series. A Series of Modern Surveys in Mathematics], vol. 61, Springer, Cham (2016)

53. Melo, M., Molcho, S., Ulirsch, M., Viviani, F., Wise, J.: Tropicalizing the universal jacobian: a logarithmic perspective. Manuscript in preparation (2020)

54. Mochizuki, S.: Foundations of $p$-adic Teichmüller theory, AMS/IP Studies in Advanced Mathematics, vol. 11. RI; International Press, Cambridge, MA, American Mathematical Society, Providence (1999)

55. Mochizuki, S.: An introduction to $p$-adic Teichmüller theory, Cohomologies $p$-adiques et applications arithmétiques (I) (Pierre, B., Jean-Marc, F., Luc, I., Kazuya, K., Michael, R. (Eds.), Astérisque, no. 278, Société mathématique de France, pp. 1-49 (en) (2002)

56. Mumford, D.: An analytic construction of degenerating curves over complete local rings. Compositio Math. 24, 129-174 (1972)

57. Möller, M., Ulirsch, M., Werner, A.: Realizability of tropical canonical divisors. J. Eur. Math. Soc. (JEMS) 23(1), 185-217 (2021)

58. Marcus, S., Wise, J.: Logarithmic compactification of the Abel-Jacobi section. arXiv:1708.04471 [math] (2017). https://doi.org/10.1112/plms.12365

59. Molcho, S., Wise, J.: The logarithmic Picard group and its tropicalization. arXiv:1807.11364 [math] (2018)

60. Ogus, A.: Lectures on Logarithmic Algebraic Geometry, Cambridge Studies in Advanced Mathematics, vol. 178. Cambridge University Press, Cambridge (2018)

61. Olsson, M.C.: Logarithmic geometry and algebraic stacks. Ann. Sci. École Norm. Sup. (4) 36(5), 747-791 (2003)

62. Piwek, M.: The formal Teichmüller space for stable Mumford curves. Arch. Math. (Basel) 60(2), 182-200 (1993)

63. Jérôme, P., Turchetti, D.: Schottky spaces and universal Mumford curves over $\mathbb{Z}$ (Manuscript in preparation) (2020)

64. Rabinoff, J.: Tropical analytic geometry, Newton polygons, and tropical intersections. Adv. Math. 229(6), 3192-3255 (2012)

65. Ranganathan, D.: Skeletons of stable maps I: rational curves in toric varieties. J. Lond. Math. Soc. (2) 95, $\mathrm{m} * 04-832$ (2017)

66. Ranganathan, D.: Logarithmic Gromov-Witten theory with expansions. arXiv:1903.09006 [math] (2019)

67. Ranganathan, D.: A note on cycles of curves in a product of pairs. arXiv:1910.00239 [math] (2019)

68. Reinecke, E.: The cohomology of the moduli space of curves at infinite level. arXiv:1911.07392 [math] (2019)

69. Ranganathan, D., Santos-Parker, K., Wise, J.: Moduli of stable maps in genus one and logarithmic geometry, I. Geom. Topol. 23(7), 3315-3366 (2019)

70. Ranganathan, D., Santos-Parker, K., Wise, J.: Moduli of stable maps in genus one and logarithmic geometry. II. Algebra Number Theory 13(8), 1765-1805 (2019)

71. Scholze, P.: On torsion in the cohomology of locally symmetric varieties. Ann. Math. 182(3), 945-1066 (2015)

72. Serre, J-P.: Trees, Springer Monographs in Mathematics, Springer-Verlag, Berlin, Translated from the French original by John Stillwell, Corrected 2nd printing of the 1980 English translation (2003)

73. Thuillier, A.: Géométrie toroïdale et géométrie analytique non archimédienne. Application au type dhomotopie de certains schémas formels, Manuscripta Math. 123(4), 381-451 (2007)

74. Ulirsch, M.: Tropical geometry of moduli spaces of weighted stable curves. J. Lond. Math. Soc. (2) 92(2), 427-450 (2015)

75. Ulirsch, M.: Functorial tropicalization of logarithmic schemes: the case of constant coefficients. Proc. Lond. Math. Soc. 114(6), 1081-1113 (2017)

76. Ulirsch, M.: Tropicalization is a non-Archimedean analytic stack quotient. Math. Res. Lett. 24(4), 1205-1237 (2017)

77. Ulirsch, M.: Non-Archimedean geometry of Artin fans. Adv. Math. 345, 346-381 (2019)

78. Viviani, F.: Tropicalizing vs compactifying the Torelli morphism. Tropical and non-Archimedean geometry, Contemp. Math., vol. 605, American Mathematical Society, Providence, RI, pp. 181-210 (2013) 
79. Yu, T.Y.: Enumeration of holomorphic cylinders in log Calabi-Yau surfaces. I. Math. Ann. 366(3-4), 1649-1675 (2016)

80. Yu, T.Y.: Gromov compactness in non-archimedean analytic geometry. J. Reine Angew. Math. 741, 179-210 (2018)

81. Yu, T.Y.: Enumeration of holomorphic cylinders in log Calabi-Yau surfaces, II: positivity, integrality and the gluing formula. Geom. Topol. 25(1), 1-46 (2021)

Publisher's Note Springer Nature remains neutral with regard to jurisdictional claims in published maps and institutional affiliations. 